\title{
A change of view: homologous recombination at single- molecule resolution
}

\author{
Kyle Kaniecki ${ }^{1}$, Luisina De Tullio ${ }^{2,3}$, and Eric C. Greene ${ }^{3}$ \\ ${ }^{1}$ Department of Genetics and Development, Columbia University, New York, New York 10032, \\ USA \\ ${ }^{2}$ Centro de Investigaciones en Química Biológica de Córdoba (CIQUIBIC), Consejo Nacional de \\ Investigaciones Científicas y Técnicas (CONICET), Departamento de Química Biológica, \\ Facultad de Ciencias Químicas, Universidad Nacional de Córdoba, Ciudad Universitaria, Córdoba \\ X5000 HUA, Argentina \\ ${ }^{3}$ Department of Biochemistry and Molecular Biophysics, Columbia University, New York, New York \\ 10032, USA
}

\begin{abstract}
Genetic recombination occurs in all organisms and is vital for genome stability. Indeed, in humans, aberrant recombination can lead to diseases such as cancer. Our understanding of homologous recombination is built upon more than a century of scientific inquiry, but achieving a more complete picture using ensemble biochemical and genetic approaches is hampered by population heterogeneity and transient recombination intermediates. Recent advances in singlemolecule and super-resolution microscopy methods help to overcome these limitations and have led to new and refined insights into recombination mechanisms, including a detailed understanding of DNA helicase function and synaptonemal complex structure. The ability to view cellular processes at single-molecule resolution promises to transform our understanding of recombination and related processes.
\end{abstract}

\begin{abstract}
Homologous recombination (HR) is a conserved pathway for catalysing the exchange of genetic information between DNA molecules and has been shown to contribute to important cellular processes such as double-strand break repair (DSBR), rescue of collapsed or stalled replication forks, horizontal gene transfer and meiosis ${ }^{1-3}$. These processes all make vital contributions to genome integrity and/or enhance genetic diversity. Furthermore, unchecked recombination can lead to gross chromosomal rearrangements, toxic intermediates that can lead to cell death, and loss of heterozygosity ${ }^{4-6}$. Indeed, mutations in the proteins that carry out HR can cause serious deleterious effects, a feature emphasized by their involvement in human diseases, including cancer ${ }^{4,7-9}$.
\end{abstract}

\footnotetext{
Correspondence to E.C.G. ecg2108@cumc.columbia.edu.

Author contributions

K.K. and L.D.T researched data for the article and wrote the initial draft of the manuscript. E.C.G. reviewed and edited the manuscript before submission and prepared the final draft.

Competing interests statement

The authors declare no competing financial interests.
} 
Early genetic studies of recombination in eukaryotes led to major theoretical advances exemplified by the Holliday model of recombination, followed by the Meselson-Radding model and later the DSBR model of recombination ${ }^{5,10,11}$. Most recently, studies of gap repair in Drosophila melanogaster led to the synthesis-dependent strand annealing (SDSA) model, which is currently considered a major pathway for both mitotic and meiotic recombination in many organisms ${ }^{12}$. These successive models have been built upon decades of research that have helped to define the molecules and pathways involved in recombination (FIG. 1; TABLE 1).

Despite the wealth of knowledge about recombination that has been generated by genetic and biochemical approaches, these methods have limitations. They cannot readily be used to access information on biochemical processes that might proceed via multiple pathways, they can overlook transient or otherwise underrepresented intermediates, and they have limited access to detailed information on dynamic reaction intermediates. These limitations are now being addressed by applying single-molecule optical microscopy and super-resolution optical microscopy to in vitro and in vivo studies of DNA recombination; these approaches can help provide detailed spatiotemporal information about heterogeneous biological reaction mechanisms.

In this Review, we discuss how single-molecule (SM) and super-resolution (SR) optical microscopy are contributing to our understanding of HR. We begin by briefly describing the basic concepts behind some of the most common SM optical methods before discussing how they are being applied to study proteins involved in HR, including motor proteins, singlestranded DNA (ssDNA)-binding proteins and the RAD51/RecA family of DNA recombinases, named after the eukaryotic DNA repair protein RAD51 and its bacterial homologue RecA. We then outline the different types of SR microscopy that have been used to study HR and highlight examples of insights these studies have provided. This Review is not intended to provide a detailed overview of $\mathrm{HR}$, nor do we provide extensive discussion of prior bulk biochemical or genetic studies; for more comprehensive descriptions of HR mechanisms and discussion of experimental approaches other than optical microscopy, we direct readers to several excellent reviews ${ }^{2-4,13-18}$.

\section{SM approaches for studying recombination}

Here, we briefly highlight some of the SM methods that have been applied to study processes related to HR; for more extensive information on SM methodologies, we refer readers to other reviews ${ }^{19-24}$. Two of the more common fluorescence microscopy methods that have been used to visualize in vitro SM reactions are widefield epi-illumination and total internal reflection microscopy (TIRFM) (FIG. 2a-d). The main difference between these two approaches is the illumination scheme. With epi-illumination, which is often used in combination with laser tweezers to capture tethered molecules of interest (FIG. 2b), all fluorescent molecules present in the sample contribute to the detected signal (FIG. 2a). By comparison, only a very small volume of the sample is illuminated in TIRFM because the illumination scheme generates an evanescent field that penetrates only $~ 100-300 \mathrm{~nm}$ into the sample chamber ${ }^{25,26}$ (FIG. 2c,d). Importantly, this approach can reduce the background signal by several orders of magnitude relative to epi-illumination (compare FIG. 2a and 
2c) ${ }^{25,26}$. Applied to recombination-related reactions, epi-illumination-based techniques and TIRFM-based techniques can be used to visualize fluorescently tagged proteins as they move along nucleic acids, to monitor the colocalization of proteins on nucleic acids or to monitor the binding and dissociation kinetics of proteins. In these instances, the information acquired from the observed molecules is usually subject to standard optical resolution limits, which are typically in the order of a few hundred nanometres, although resolution in the order of $\sim 1$ to tens of nanometres can be obtained by mathematical treatment of the resulting data to identify the locations of individual fluorescent spots ${ }^{27}$. However, much higher spatial resolution can be obtained by measuring fluorescence resonance energy transfer (FRET) between biomolecules that have been appropriately labelled with donor and acceptor fluorophores ${ }^{28,29}$. The distance regime accessible to FRET studies is in the order of $\sim 10-50$ $\AA$, which is comparable to the dimensions of many proteins and makes it an ideal technique for probing biomolecular associations or structural changes that occur within these distances $^{28,29}$. Single-molecule FRET (smFRET), most commonly used in combination with TIRFM, has become one of the most widely used methods within the field of SM research ${ }^{19,21,30}$ (FIG. 2e). However, as with any FRET-based study, its success is dependent on judicious selection of sites for dye attachment, which will vary according to the system being studied and the specific question being addressed.

Importantly, all the SM optical imaging methods described here require that the molecules under observation are tethered to a surface. For instance, to manipulate molecules using an optical trap, the molecules must be anchored to the surface of a bead (FIG. 2b), whereas with TIRFM, the molecules can be detected only if they are within the evanescent field, which is located at the slide surface or coverslip surface (FIG. 2c,d). These attachments are often made through biotin-streptavidin or digoxigenin-antibody linkages. Surfaces must also be blocked to prevent nonspecific adsorption of the molecules of interest, which might compromise their biological activities. Beads are often blocked with a nonspecific protein, such as bovine serum albumin (BSA). Microscope slides can also be blocked with BSA, but another commonly used method is to chemically modify the glass surface with a layer of polyethylene glycol (FIG. 2e). With DNA curtains, nonspecific interactions are minimized by coating the surface of the microscope slide with a lipid bilayer ${ }^{31,32}$ (FIG. 2f).

\section{SM-based insights into recombination}

SM optical techniques are ideal for probing dynamic spatiotemporal processes during HR that cannot be accessed through traditional experimental approaches. We discuss their application to motor proteins, which were among the first HR proteins to be studied by SM techniques; proteins involved in assembling the presynaptic complex, namely, the ssDNAbinding proteins, which are among the first proteins to arrive at processed double-strand breaks (DSBs); and the RAD51/RecA family of DNA recombinases, which catalyse the DNA pairing and strand invasion reactions (FIG. 1).

\section{Motor proteins involved in DNA recombination}

DNA motor proteins play a wide range of roles in all processes related to nucleic acid metabolism ${ }^{33-35}$, including $\mathrm{HR}^{36-44}$ (TABLE 1). In general, these proteins use chemo- 
mechanical energy derived from ATP hydrolysis to unwind nucleic acid duplexes or to displace proteins from nucleic acids ${ }^{36-44}$. The groundbreaking work on the Escherichia coli RecBCD motor protein complex represents the earliest examples of SM studies for any protein complex involved in HR. Biochemical and genetic studies had established that RecBCD processes DSBs during HR to generate long ssDNA overhangs that can then serve as assembly sites for the RecA presynaptic complex ${ }^{45-47}$. RecBCD comprises two motors, the $3^{\prime}-5^{\prime}$ superfamily $1 \mathrm{~A}$ helicase RecBCD enzyme subunit RecB and the $5^{\prime}-3^{\prime}$ superfamily 1B helicase RecBCD enzyme subunit RecD ${ }^{34}$. RecBCD enzyme subunit RecC holds the complex together, helps to separate the DNA strands and coordinates the response to crossover hotspot instigator sites (chi sites), which together result in $3^{\prime}$ ssDNA overhangs that serve as substrates for assembly of the RecA presynaptic complex ${ }^{45,47}$ (FIG. 1). Despite this wealth of knowledge, many aspects of RecBCD activity and regulation remained unknown owing to the difficulty of analysing heterogeneous populations of molecules by traditional ensemble approaches. Specifically, it was not known how quickly RecBCD was able to move along DNA, how far it could go before falling off the DNA and what happened to the protein complex when it encountered a chi site. To overcome these problems, single molecules of RecBCD acting on DNA were visualized using widefield epifluorescence microscopy ${ }^{48}$. Fluorescently labelled DNA molecules were attached to polystyrene beads and captured within an optical trap (FIG. 2b), RecBCD was loaded onto the DNA, reactions were initiated by moving the DNA into a stream of buffer containing ATP, and time-lapse images were collected. Analysis of the data showed that RecBCD could travel along the DNA at velocities often exceeding $1,000 \mathrm{bp} \mathrm{s}^{-1}$ (at $37^{\circ} \mathrm{C}$ ) and for distances of tens of kilobases ${ }^{48}$. Remarkably, RecBCD was later shown to briefly pause at chi sites before resuming translocation at roughly half of its initial velocity ${ }^{49}$. This study also revealed that when powered by RecD, the lead motor before chi recognition, the RecBCD complex travels at a velocity of $\sim 600 \mathrm{bp} \mathrm{s}^{-1}$ (at room temperature), whereas the complex moves at $\sim 300 \mathrm{bp} \mathrm{s}$ ${ }^{-1}$ when powered by $\operatorname{RecB}^{49}$. Chi recognition causes RecD to stop translocating, at which point RecB takes over as the lead motor. Thus, the pause at chi sites is a consequence of the differential velocities of RecD and RecB and reflects the time it takes for the slower RecB motor to catch up to RecD. Chi recognition also alters RecB nuclease activity such that it selectively degrades only one ssDNA strand and allows productive DSB processing, although the precise underlying mechanism remains uncertain ${ }^{45,47}$. Together, these SM imaging studies have led to a remarkably detailed understanding of RecBCD regulation that would not have been possible using only traditional approaches ${ }^{45}$.

The level of detail that can be obtained from SM methods is exemplified by a series of smFRET studies that documented the properties of four structurally homologous helicase proteins: ATP-dependent DNA helicase Rep and DNA helicase II (UvrD) from E. coli, ATPdependent DNA helicase PcrA from Staphylococcus aureus and ATP-dependent helicase Srs 2 from Saccharomyces cerevisiae ${ }^{50-52}$. Genetic and biochemical studies have shown that $S$. cerevisiae Srs2 removes DNA repair protein Rad51 from ssDNA, thus acting as a negative regulator of genetic recombination ${ }^{39,42,53,54}$, and similar properties have been attributed to Rep, PcrA, UvrD and many other HR-related helicases ${ }^{37,42,55-58}$. In these experiments, smFRET signals were detected with TIRFM and used to measure the movement of the helicases along DNA (FIG. 2e). In the first study, a dsDNA fragment with a $3^{\prime}$ ssDNA 
overhang was tagged with a fluorescence acceptor dye at the ssDNA-dsDNA junction. The fluorescence donor dye was coupled to Rep through site-specific labelling of a cysteine residue. This setup allowed the distance between Rep and the ssDNA-dsDNA junction to be measured using smFRET ${ }^{51}$ (FIG. 2e). As expected, addition of the helicase Rep resulted in an increase in the smFRET signal as the helicase moved along the ssDNA towards the ssDNA-dsDNA junction (FIG. 2e). This study also detected unusual cyclical changes in the smFRET signals that were interpreted as repeated translocation events, which suggested that the motor domains of the helicases dissociated from the DNA upon reaching the ssDNAdsDNA junction (FIG. 2e). However, some part of the protein retained in contact with the ssDNA to enable the process to be repeated for many cycles before the helicase dissociated into free solution. Notably, this type of cycling behaviour can be observed only by SM imaging. Similar smFRET studies have now shown that Srs2 and PcrA remove Rad51 and RecA filaments from ssDNA, respectively, and have suggested that the repetitive shuttling of these helicases prevents aberrant recombination by clearing these recombinases from ssDNA $^{51,52,55}$.

The part played by conformational changes and oligomerization in the regulation of helicases has been difficult to measure with ensemble techniques because of the transient nature of conformational changes and the population heterogeneity associated with oligomerization $33,59,60$. Structural studies had predicted that the helicases Rep, UvrD and PcrA might undergo large conformational changes - all three proteins were thought to transition between 'open' and 'closed' helicase conformations via an $\sim 150^{\circ}$ rotation of the $2 \mathrm{~B}$ domain relative to the rest of the protein ${ }^{61-63}$. Recent SM studies have investigated how structure and function affect helicase activity using elegant combinations of smFRET to probe protein conformation and force-based measurements to probe DNA unwinding ${ }^{59,60}$. In the first of these studies, Rep and PcrA protein variants were generated that contained pairs of engineered cysteines that could be used to 'lock' the proteins into the closed conformation ${ }^{59}$. These proteins were then probed for activity in an smFRET assay in which the proteins were immobilized on a slide surface and a DNA substrate containing an $18 \mathrm{bp}$ duplex region and a $3^{\prime}$ 20-nucleotide ssDNA tail was added ${ }^{59}$. A Cy3 donor was attached to the $3^{\prime}$ ssDNA end of the substrate, and a Cy5 acceptor was attached to the opposite end of the duplex. Partial unwinding of the duplex led to an increased smFRET signal as the donor and acceptor dyes approached one another, and complete unwinding resulted in a loss of smFRET owing to displacement of a DNA strand ${ }^{59}$. Interestingly, this study found that Rep or PcrA locked in the closed conformation exhibited greater processivity than the wild-type enzymes - for instance, $95 \%$ of the Rep molecules locked into the closed conformation could completely unwind a $4 \mathrm{~kb}$ region of duplex DNA, whereas only $7 \%$ of the wild-type Rep could fully unwind the same region of duplex DNA. This result suggested that reorientation of the $2 \mathrm{~B}$ domain into the open conformation restricts the ability of Rep and PcrA to unwind long stretches of dsDNA ${ }^{59}$.

In a different study, smFRET analysis of UvrD labelled with donor and acceptor dye pairs on the $2 \mathrm{~B}$ and $1 \mathrm{~A}$ domains revealed the existence of two distinct activities, which were termed 'frustrated' and 'long-distance' unwinding ${ }^{60}$. Frustrated activity was characterized by repetitive cycles of unwinding and rezipping over distances shorter than $20 \mathrm{bp}$, whereas long-distance activity was less repetitive and allowed for unwinding of tracts of dsDNA 
longer than $20 \mathrm{bp}$. Counting the number of fluorescent UvrD molecules present on each DNA revealed that the frustrated and long-distance behaviours correlated with one and two UvrD proteins, respectively. This study also established an smFRET assay that was able to report on whether a particular molecule of UvrD was in the open or closed state $e^{60}$. These findings led to a model in which UvrD (and related helicases) can switch directions by transiently sampling the open configuration: the $2 \mathrm{~B}$ domain anchors the helicase to the DNA so that the motor domains can dissociate from one strand and then rebind the second strand to move in the opposite direction ${ }^{60}$. The frequency of open-to-closed transitions dictates how much DNA a given enzyme can unwind before switching directions and rewinding the same substrate; this frequency is influenced by the number of molecules present, which explains why two UvrD proteins acting in concert exhibit long-distance unwinding events ${ }^{60}$.

\section{Single-stranded binding proteins}

All recombination reactions involve an ssDNA intermediate, which is rapidly coated by essential proteins - single-stranded DNA-binding protein (SSB) in bacteria and replication protein A (RPA) in eukaryotes ${ }^{64,65}$. Biochemical and genetic studies have shown that SSB and RPA protect the ssDNA from nucleases, eliminate secondary structure to enable recombinase loading, serve as DNA damage signalling complexes and promote recruitment of downstream factors ${ }^{64,66-71}$ (FIG. 1). Although the need for these proteins in all aspects of DNA metabolism is well established, we still have a fairly poor understanding of their ssDNA-binding properties, in particular their dynamic behaviour on ssDNA and the mechanisms that allow them to be replaced by downstream factors during the repair of DNA damage. The development of ssDNA curtains (FIG. 2f) afforded the opportunity to study the properties of RPA on long ssDNA substrates ${ }^{31,72}$. These experiments have revealed that GFP-tagged RPA can bind tightly to ssDNA and that essentially no protein dissociation occurs within a 2-hour observation window when free RPA was absent from solution ${ }^{73-75}$. Introducing free RPA resulted in rapid turnover of the DNA-bound proteins, suggesting RPA was replaced through a concentration-dependent mechanism referred to as facilitated dissociation ${ }^{73}$. Facilitated dissociation is a mass action process that can take place when a protein is capable of interacting with its ligand through multiple contacts (for example, the three ssDNA-binding domains of RPA). Transient disruption of any single contact is not sufficient to allow the protein to release its ligand, and in the absence of competing interactions, the disrupted contact can rapidly reform. However, when free proteins are present, they can rapidly engage any sites within the ligand that are transiently released by the bound proteins, which in turn reduces the probability of rebinding. The advantage of facilitated dissociation is that it offers the potential for extremely tight binding interactions but still allows rapid displacement of the bound proteins ${ }^{44,45}$. Facilitated dissociation has also been reported for several other DNA-binding proteins ${ }^{76,77}$, suggesting that this process is more common in biological systems than is currently appreciated ${ }^{78,79}$.

Other aspects of RPA and SSB behaviour on ssDNA have been studied using SM approaches. For instance, smFRET studies have shown that SSB can diffuse along ssDNA, and this process may be involved in the displacement of SSB from ssDNA during the assembly of the RecA presynaptic complex ${ }^{80}$. SSB can even be pushed along ssDNA through the action of the E. coli helicases Rep and UvrD, which suggests a role for these 
helicases in assisting the reorganization or removal of SSB from DNA repair intermediates ${ }^{81}$. A TIRFM study looking at time-resolved widefield images of SSB bound to ssDNA revealed that SSB can form reversible long-range contacts that can lead to extensive condensation of SSB-bound ssDNA intermediates ${ }^{82}$. Finally, DNA curtain studies have shown that RPA can downregulate the processivity of the DNA-end-processing nuclease exonuclease 1 (EXO1), which suggests an unforeseen role for RPA in regulating EXO1 activities $^{83}$. These studies highlight some of the new discoveries that have been made through the use of SM optical microscopy, in particular, that SSB and RPA are highly dynamic complexes that are capable of moving, exchanging and forming higher-order structures.

\section{Dynamics of the presynaptic complex}

The presynaptic complex is a crucial recombination intermediate formed when RPA is replaced by RAD51 (or meiotic recombination protein DMC1/LIM15 homologue (DMC1)) in eukaryotes or when SSB is replaced by RecA in bacteria (FIG. 1). Decades of biochemical and genetic studies have led to a detailed understanding of biochemical properties of RecA and Rad51 nucleoprotein filaments $15,17,18,56,70,84$. However, we still do not fully understand how these filaments are assembled or how they search the genome for homologous sequences, discriminate between homologous and non-homologous sequences and promote the strand-pairing reactions necessary for genetic recombination. We also lack an understanding of the precise complement, stoichiometry and spatial organization of the HR accessory factors that must interact with the presynaptic complex to promote its stability and activities. SM imaging methods offer the potential to help fill these gaps in our understanding.

An early SM investigation into presynaptic complex dynamics used smFRET to observe assembly of RecA filaments on short ssDNA overhangs ${ }^{85}$ (FIG. 3a). This study suggested that $\sim 5$ monomers of RecA are required to form a stable nucleation point for filament assembly and that subsequent extension occurs in single monomer units. Most surprisingly, these experiments suggested that filament extension occurs in both the $5^{\prime}-3^{\prime}$ and $3^{\prime}-5^{\prime}$ directions. However, monomer binding occurred more rapidly at the $3^{\prime}$ end of the growing filaments, while monomer dissociation occurred at similar rates at either end, leading to more rapid net extension in the $5^{\prime}-3^{\prime}$ direction ${ }^{85}$.

Bidirectional growth of RecA filaments was also observed in studies using fluorescently tagged RecA and a three -channel laminar flow system to control the assembly of RecA filaments on bead-tethered dsDNA molecules ${ }^{23,86,87}$ (FIG. 3b). The centre channel was used for observation by epifluorescence microscopy, whereas an adjacent channel was used to capture the bead-DNA complex in an optical trap. The captured DNA was then 'dipped' into the third flow stream containing fluorescent RecA and moved back into the central channel for observation. This dipping allowed the authors to precisely control the timing with which the RecA and the DNA were mixed together and to control the amount of RecA that bound to the DNA based upon the length of time that the DNA remained in the RecA channel. The resulting snapshots revealed that filament assembly occurs through a ratelimiting nucleation step involving the initial binding of $\sim 4-5$ RecA proteins. Nucleation was

Nat Rev Genet. Author manuscript; available in PMC 2018 October 01. 
followed by a rapid growth phase in which the filaments could extend in both the $5^{\prime}-3^{\prime}$ and $3^{\prime}-5^{\prime}$ directions.

Building upon this work, another study used TIRFM to look at long molecules $(\sim 48,000$ nucleotides) of SSB-coated ssDNA that were anchored to a flowcell surface ${ }^{88}$. Use of a clever two-colour labelling strategy, involving RecA labelled with either Cy3 or fluorescein, allowed the authors to distinguish between nucleation and growth within the same filaments (FIG. 3c), which provided a clear demonstration that growth was bidirectional but occurred roughly twice as quickly in the $5^{\prime}-3^{\prime}$ direction compared with the $3^{\prime}-5^{\prime}$ direction ${ }^{88}$. Next, the role of the RecFOR proteins, which help promote RecA assembly on ssDNA ${ }^{89,90}$, was investigated by SM observation ${ }^{88}$. RecFOR caused the tethered SSB-ssDNA to nonspecifically absorb to the slide surface, so these measurements relied upon epifluorescence observations of a bead-tethered DNA, which enabled the DNA to be held away from the slide surface ${ }^{88}$. These experiments showed that in the presence of RecFOR, the frequency of RecA nucleation increased, as did the rate of filament growth, suggesting that RecA was more readily able to replace SSB when RecFOR was present ${ }^{88}$. Together with previous bulk biochemical studies ${ }^{15}$, these observations support a model in which RecFOR promotes RecA presynaptic complex assembly by weakening contacts between SSB and ssDNA, perhaps by partially unwrapping SSB from the ssDNA ${ }^{88}$.

DNA curtains have also been used to study the assembly and stability of the presynaptic complex, using a GFP-RPA fusion protein as a readout for protein occupancy on the $\operatorname{ssDNA}^{72-74,91}$; the absence of GFP fluorescence indicates that the unlabelled (or dark) protein of interest has displaced GFP-RPA from the ssDNA (FIG. 3d). One notable advantage of this labelling strategy is that it avoids the need to prepare fluorescently tagged recombinases, which have proved difficult to generate without substantially compromising biochemical activities. This DNA curtain approach has been applied to $E$. coli RecA, $S$. cerevisiae and human RAD51 and DMC1 and Caenorhabditis elegans RAD-51 (REFS 7275,91-95). Collectively, these studies have confirmed that human RAD51 exhibits bidirectional, ATP-dependent filament assembly ${ }^{75}$; that the yeast mediator protein DNA repair and recombination protein Rad52 can bind extensively to an assembled presynaptic complex, which highlights potential roles for Rad52 after presynaptic complex assembly ${ }^{91}$; and that the $C$. elegans RAD51-like protein 1 (RFS-1)-RFS-1 interacting partner (RIP-1) complex (a paralogue of RAD51) stabilizes filaments by preventing RAD-51 dissociation even in the absence of ATP, which highlights the dramatic effect that the RAD51 paralogues can have on presynaptic complex stability ${ }^{95}$.

When RAD51 or RecA bind to DNA, they stretch it by $\sim 50 \%$ relative to the contour length of B-DNA ${ }^{84,96}$. To explore the possibility of a relationship between DNA tension and filament stability, an epifluorescence microscope equipped with dual optical traps ${ }^{97-100}$ was used to visualize fluorescent RAD51 filaments while simultaneously stretching the dsDNA; these studies demonstrated that increased tension on the DNA prevented protein dissociation and thereby increased filament stability ${ }^{97}$. Surprisingly, when tension was released, filament disassembly occurred in bursts interspersed by long pauses. Analysis of the data revealed a clear relationship between extension of the presynaptic complex and the dissociation of RAD51 ${ }^{97}$. Moreover, the patterns of protein dissociation that were observed led to a model 
in which RAD51 could hydrolyse ATP anywhere within the stretched filament, but dissociation of the resulting ADP-bound proteins could occur only at the filament ends; ATP-bound proteins were unable to dissociate from filaments regardless of their position ${ }^{97}$. Thus, the bursts of RAD51 dissociation corresponded to filaments with ADP-bound proteins at their ends, whereas pauses occurred when ATP-bound proteins were at the filament ends.

Together, the studies highlighted above are beginning to yield new insights into the behaviours of the presynaptic complex, but much remains to be discovered. Future investigations of the presynaptic complex with SM approaches might help to address remaining questions about protein composition, protein distributions and reaction mechanisms.

\section{The homology search and strand invasion}

The presynaptic filament has a number of vital roles in genetic recombination. First, it must locate a homologous dsDNA sequence present elsewhere in the genome in a process that is referred to as the homology search. Then, it must promote strand invasion by catalysing Watson- Crick pairing interactions between the presynaptic ssDNA and the complementary strand within the homologous dsDNA sequence while also displacing the noncomplementary strand to generate a D-loop structure ${ }^{15,17}$. Both the homology search and strand invasion are highly dynamic processes that are anticipated to proceed through a heterogeneous and asynchronous set of intermediates, which makes it difficult to study the molecular details with biochemical and genetic methods.

The homology search has been the subject of a number of in-depth reviews ${ }^{86,101-103}$, and thus here we only briefly highlight the key areas where progress has been made using a combination of bulk biochemical studies and SM studies. For instance, these types of studies are beginning to present a more unified picture of the basic principles that guide sequence alignment during the homology search. They have shown that the process involves a combination of intersegmental transfer ${ }^{104,105}$, short-distance 1-dimensional sliding ${ }^{106}$ and a length-dependent recognition mechanism that reduces search complexity by allowing the search process to focus on tracts of microhomology longer than eight nucleotides, which have a high probability of being the correct target ${ }^{94,107-110}$. Other studies suggest that differential extension of the presynaptic ssDNA relative to the incoming dsDNA gives rise to differential levels of tension between the two DNA molecules, which promotes more rapid turnover of non-homologous pairing interactions ${ }^{111}$. However, many unanswered questions remain, including how recombination accessory proteins, such as the motor proteins DNA repair and recombination protein Rad54 and DNA repair and recombination protein Rdh54 (REFS 3,4,15,16), influence the search process and how the search takes place within chromatin.

Strand invasion has been more difficult to study using SM optical studies than other aspects of recombination, largely owing to the rapid reaction rates and the small length scales involved in this process. Nevertheless, an assay that combines TIRFM and smFRET has been used to visualize the capture of homologous dsDNA fragments by a RecA presynaptic complex anchored to a slide surface ${ }^{112}$. Using this approach, strand exchange led to a high FRET signal between a Cy5 acceptor positioned on the presynaptic complex and a Cy3 
donor placed on the incoming dsDNA. However, there was a considerable delay, on the order of $\sim 120$ milliseconds, between the initial binding of the dsDNA fragment and the appearance of the FRET signal, which was attributed to the time required to complete strand invasion. Subsequent analysis of the delay time distributions suggested that strand exchange took place in 3-nucleotide steps ${ }^{112}$. Similar stepping behaviours during strand invasion have been observed using ssDNA curtain assays ${ }^{92-94}$. In these studies, D-loop intermediates were generated by incubating RecA, RAD51 or DMC1 presynaptic complexes with fluorescently tagged dsDNA fragments containing an internal microhomology of 8-15 nucleotides in length, which was complementary to the ssDNA. The resulting lifetime changes occurred in 3 -nucleotide increments, consistent with the notion that strand exchange intermediates are stabilized by the RAD51/RecA family members in 3-nucleotide steps ${ }^{92-94}$. Moreover, the energetic signature of the stepping behaviour was conserved for RecA, RAD51 and DMC1, indicating that this behaviour appears to be broadly conserved among members of the RAD51/RecA family of DNA recombinases. However, a caveat of both approaches is that they do not directly 'see' individual strand exchange steps as they are taking place. Rather, the existence of the 3-nucleotide steps is inferred from either a model-dependent analysis of kinetic data ${ }^{112}$ or from the stability of the resulting strand exchange products ${ }^{92-94}$. Direct observation of the steps themselves remains a goal of future technical developments.

\section{SR approaches for studying recombination}

Until recently, optical microscopy suffered from a fundamental limit in spatial resolution owing to the wave nature of visible light. This phenomenon, first described as the Abbe limit in 1873 and quantified by the Rayleigh criterion, means the maximum achievable resolution for discrete fluorescent sources is equal to one-half of the emission wavelength of the fluorophores under observation ( $250 \mathrm{~nm}$ for GFP, which has an emission wavelength of $509 \mathrm{~nm})^{113-116}$. Unfortunately, this value remains two orders of magnitude above the size regime that is relevant to many molecular interactions and subcellular structures - in other words, the smallest distance that can be resolved using fluorescent labels and traditional imaging techniques is usually much greater than the distances relevant to many biological processes. However, new imaging techniques are now beginning to leverage advances in fluorophore photophysics and image analysis to enable measurements within spatial regimes that were previously inaccessible ${ }^{114-119}$. These new imaging techniques include stochastic optical reconstruction microscopy (STORM) ${ }^{120-122}$, photo-activated localization microscopy (PALM) ${ }^{123-125}$, stimulated emission depletion (STED) microscopy ${ }^{126-128}$ and structured illumination microscopy (SIM) $)^{129-131}$.

STORM and PALM both use the same general principles for generating SR images, namely, they take advantage of the fact that single fluorophores can be localized with great precision by using relatively simple equations to calculate the centroid position of a fluorescent spot representing a single fluorescent dye ${ }^{113-115}$. However, these localization procedures work only if the fluorescent dyes are relatively far apart from one another. Thus, a key feature of these methods is that not all the fluorophores present in a sample are visualized at once. Instead, a series of images are collected in which only a small subset of the fluorophores present on a labelled subcellular structure are activated at any given time, and a complete composite picture is then generated by integrating all the fluorophore position information 
derived from the image series ${ }^{113-115}$ (FIG. 4A). In both PALM and STORM, the fluorophores present in the sample are driven between 'light' or 'on' (that is, visible) and 'dark' or 'off ' (that is, not visible) states using variations in excitation wavelength, which enables only a small number of the total fluorophores present to be excited at a given time. The main difference between the two methods is that PALM uses engineered versions of GFP that can be switched on and off, whereas STORM typically relies upon the use of antibodies coupled to organic fluorophores ${ }^{113-115}$.

STED shares one common feature with PALM and STORM; namely, it relies upon the excitation of only a small subset of the fluorophores present in a given image at any given time to generate an SR image. However, in STED, this is accomplished by using a focused doughnut-shaped light beam to switch fluorophores between 'on' and 'off' states at defined spatial coordinates - only those fluorophores present in the very centre of the doughnut are 'on', and the remaining fluorophores are switched 'off ' (REFS 113-115) (FIG. 4B). The doughnut-shaped beam is then scanned across the sample to generate a complete image, and the resolution of the resulting image is defined by the size of the doughnut centre. SIM also uses patterned light for sample illumination, but in this case, the entire sample is illuminated with a series of striped light patterns ${ }^{113-115}$ (FIG. 4C). The recorded images are then computationally processed to produce a mathematically reconstructed image that has about twice the resolution of normal fluorescence microscopy.

STORM, PALM and STED excel at imaging fixed cells or chromosome spreads, but they are currently more difficult to use for live-cell imaging, especially for biological processes that occur rapidly, because these methods require the time-consuming collection of many successive images to build a composite picture of the structures of interest ${ }^{113-115}$. SIM offers great promise for live-cell imaging because images can be captured at higher acquisition rates, currently on the order of several seconds, albeit with lower spatial resolution than that offered by STORM, PALM and STED ${ }^{113-115}$.

\section{SR-based insights into recombination}

SR approaches are being actively applied by a number of laboratories to study the nucleoprotein structures that participate in meiosis and meiotic recombination. Although there are currently only a few such studies in the published literature, we anticipate this number to increase in the coming years as their potential for studying HR becomes more widely appreciated.

\section{Organization of the synaptonemal complex}

Meiosis is characterized by a single round of replication followed by two rounds of cell division to yield haploid gametes from a diploid precursor ${ }^{132-136}$ (FIG. 5a). Meiotic recombination is required for chromosome segregation, and it also generates new combinations of parental alleles. During meiosis, the endonuclease meiotic recombination protein SPO11 initiates programmed DSBs, which are then repaired by HR between homologues. This preference for repair using the homologue, instead of the sister, is referred to as 'homologue bias', but its underlying mechanisms remain unknown ${ }^{132-136}$. Importantly, the earliest stages of meiotic recombination coincide with the formation of a meiosis- 
specific chromo some structure referred to as the synaptonemal complex ${ }^{132-136}$ (FIG. 5b). The synaptonemal complex comprises two lateral elements, which serve as attachment points for homologous chromosomes, and the DNA, which is arranged as a linear array of loops tethered to the lateral elements ${ }^{132-136}$ (FIG. 5b). The lateral elements are held together by a central axis region that can be further subdivided into a single central element, which runs midway between the two lateral elements, and transversal filaments, which link the central and lateral elements ${ }^{132-136}$ (FIG. 5b). This structural organization of the synaptonemal complex is broadly conserved among eukaryotes, although the protein components tend to be more divergent ${ }^{132-136}$. Much of our understanding of the synaptonemal complex has come from genetic, electron microscopy and conventional optical microscopy studies ${ }^{132-136}$. However, we still have a limited understanding of its 3dimensional organization, a limited knowledge of the detailed spatial distribution and organization of proteins within it, and a very poor understanding of how its unique structural organization contributes to the homologue bias that is a defining characteristic of meiotic recombination ${ }^{132-136}$.

Questions relating to the structure and function of the synaptonemal complex can potentially be addressed using new SR imaging methods. For instance, the lateral and central elements cannot be resolved by conventional fluorescence microscopy, but they have been resolved by STORM $^{137,138}$. In one initial study, fluorescently tagged antibodies targeting structurespecific proteins (synaptonemal complex protein 2 (SYCP2; also known as SCP2) and SYCP3 (also known as SCP3) in the lateral elements and SYCP1 (also known as SCP1) in the transversal filaments) were used to generate STORM images of the synaptonemal complex in mammalian chromosome spreads ${ }^{137}$ (FIG. 5c). Antibodies that targeted either the N-terminal domain or the C-terminal domain of SYCP1 enabled each domain to be visualized independently and provided confirmation that the C-terminus of SYCP1 localizes with the lateral elements, whereas its N-terminus localizes with the central elements ${ }^{137}$ (FIG. 5c). In another study, STORM was used with fluorescently tagged antibodies directed against modified histones to look at the distributions of epigenetic markers within the synaptonemal complex, revealing distinctive patterns of histone modifications ${ }^{138}$ (FIG. 5d). For instance, the repressive histone modification $\mathrm{H} 3 \mathrm{~K} 27 \mathrm{me} 3$ was found in periodic clusters positioned at $500 \mathrm{~nm}$ intervals, whereas the centromeric histone modification $\mathrm{H} 3 \mathrm{~K} 9 \mathrm{me} 3$ was located at one end of the synaptonemal complex, consistent with the known location of mouse centromeres. In addition, $\mathrm{H} 3 \mathrm{~K} 4 \mathrm{me} 3$, a histone modification associated with transcriptionally active chromatin, was found to be arranged in a radial 'hair-like' pattern that emanated outwards from the synaptonemal complex and that was suggestive of the chromatin loops expected to project outwards from the synaptonemal complex (FIG. 5b,d). Together, these studies have yielded remarkably detailed 3-dimensional pictures of the synaptonemal complex, and a side-by-side comparison of images collected by conventional microscopy with those collected by STORM provides an intuitive understanding of the degree to which spatial resolution is improved with SR techniques ${ }^{137,138}$ (FIG. 5c,d).

Two very recent studies have also exploited SR imaging to further probe protein organization in the synaptonemal complex ${ }^{139,140}$. In the first of these studies, researchers labelled several protein components of the $C$. elegans synaptonemal complex using either fluorescent antibodies or GFP-fusion proteins and then visualized these proteins in intact 
gonads by either STORM or PALM ${ }^{140}$. This strategy took advantage of the fact that chromosomes in $C$. elegans gonads tend to be positioned towards the nuclear envelope, thus allowing them to be illuminated by a TIRFM-like technique ${ }^{140}$. For this study, the labelled proteins included meiosis-specific HORMA domain proteins (HIM-3 and its paralogues HTP 1, HTP-2 and HTP-3) and the structural maintenance of chromosomes (SMC) cohesin complexes, including the meiosis-specific kleisin subunits meiotic recombination protein REC-8, COH-3 and COH-4 (REF. 140). The resulting SR images revealed the organization of these protein components in exquisite detail and suggested a model in which HORMA domain proteins tether cohesin-bound sister chromatids to the central regions, with REC-8containing cohesin attaching to each sister chromatid and COH-3 cohesin forming a boundary between sisters ${ }^{140}$. In the second study, researchers used SIM to visualize the protein components of the $D$. melanogaster synaptonemal complex ${ }^{139}$. In this case, the samples were fixed and expanded fourfold using a specialized tissue expansion technique originally developed for electron microscopy ${ }^{139}$. Several protein components of the synaptonemal complex were labelled using antibodies, including $\mathrm{C}(2) \mathrm{M}$, which localizes to the outer edge of the central region, and C(3)G, Corolla and CONA, which are all components that lie near the middle of the D. melanogaster central element ${ }^{139}$. A key finding from this study, which was consistent with the protein organization observed in $C$. elegans $^{140}$, was that the synaptonemal complex seemed to be organized into two distinct layers, suggesting that each sister chromatid is maintained within a spatially distinct volume ${ }^{139}$ (FIG. 5e). Although speculative, this model could help to explain why meiotic recombination is biased towards pairing interactions between homologues instead of sisters $^{139,140}$.

\section{DMC1 and the presynaptic complex}

Most eukaryotes have evolved a meiosis-specific recombinase, DMC1, and both it and RAD51 are required for progression through meiosis ${ }^{132,141,142}$. It is not understood why this is the case, nor is it understood how RAD51 and DMC1 are organized with the presynaptic complex during meiosis or how this organization might contribute to the unique requirements of meiotic recombination. Conventional microscopy studies had suggested the existence of partially offset RAD51 and DMC1 co-foci ${ }^{141-144}$, which led to the proposal of an asymmetric loading model in which RAD51-only filaments formed on one end of a meiotic DSB and DMC1-only filaments formed on the other end of the same DSB ${ }^{143-145}$. However, these studies lacked the spatial resolution necessary to confirm the model, and the model itself was at odds with a number of bio chemical and genetic studies ${ }^{146}$. To help resolve these issues, fluorescently tagged antibodies directed against RAD51 and DMC1 were used with STORM to examine the organization of the presynaptic filaments at both ends of a meiotic DSB ${ }^{146}$. The results showed that the two ends of the DSB were separated from one another by $\sim 400 \mathrm{~nm}$, and it was suggested that this distance reflected structural constraints imposed by the tethered-loop architecture of the synaptonemal complex ${ }^{146}$. Notably, RAD51 and DMC1 co-occupied both ends of the meiotic DSBs, arguing against the asymmetric loading model. These filaments containing both RAD51 and DMC1 were quite small and typically comprised just $\sim 30$ recombinase monomers, which is sufficient to cover $\sim 100$ nucleotides of ssDNA and is surprising given that the ssDNA overhangs were on the order of $\sim 800$ nucleotides in length ${ }^{141,146}$. In addition to revealing new insights into the 
presynaptic complex, this important finding illustrates how poorly we understand the protein stoichiometry and composition of the presynaptic complex and raises questions as to what other proteins are present on the ssDNA, how they are arranged and why they are there ${ }^{141}$.

\section{Little bundles of RecA}

In all the studies cited above, the cells and subcellular structures had to be fixed to ensure good imaging. An advantage of SIM is that it can be readily applied to living cells, which is essential for understanding how subcellular structures evolve in time during the completion of a biological process. Live-cell SIM has recently been applied to study RecA during DNA recombination in E. coli. During replication of the circular E. coli genome, the resulting sister chromatids move away from one another, posing the questions of whether and how recombination could occur after the homologous sequences have moved to distant cellular compartments. When induced DSBs were tracked in living cells at different time points after replication initiation, it was found that distant sister chromatids could pair even after reaching opposite ends of the cell ${ }^{147}$. Furthermore, fluorescent RecA colocalized with the DSB and then polymerized into long filamentous structures that were termed RecA 'bundles' (FIG. 6a,b). The bundles often spanned the entire length of the cell, did not have appreciable turnover as measured with fluorescence recovery after photo-bleaching (FRAP) and resulted in rapid sister pairing $~ 50$ minutes after DSB induction. SIM images revealed that the bundles were positioned between the inner membrane and the nucleoid and that they comprised a thick immobile central region flanked on either end by thinner, more mobile projections (FIG. 6b-d). In future work, it will be important to determine how these bundles of RecA contribute to recombination and whether similar dynamic structures might exist in other organisms.

Together, these early SR studies provide detailed new insights into the nucleoprotein complexes that participate in HR and serve as important foundations for future research. In principle, similar approaches could be applied to many other subcellular structures, such as DNA repair foci. It has been known for many years that repair foci demarcate the intracellular locations of recombination intermediates ${ }^{142,148}$, but we know very little about the precise organization and dynamic properties of the proteins, protein complexes and nucleic acid structures that form these foci ${ }^{148}$. A recent study using STED microscopy to study repair foci induced by ionizing radiation ${ }^{149}$ demonstrates that SR imaging has the potential to provide a detailed picture of how the nucleoprotein complexes involved in recombination are organized in living cells.

\section{Conclusions and future perspectives}

In this Review, we highlight how SM and SR optical microscopy techniques have contributed to our understanding of HR. In vitro SM studies combine high spatial and temporal resolution with the power of reductionist biochemistry to yield detailed new insights into reaction mechanisms. SR microscopy is a rapidly evolving area of research that offers exceptional spatial resolution, which provides new insight into how recombination proteins and related nucleic acid structures are organized in living cells. It is, of course, important to recognize that these microscopy techniques will never operate in isolation, and

Nat Rev Genet. Author manuscript; available in PMC 2018 October 01. 
they will always be guided by both genetic studies and bulk biochemical analysis.

Nevertheless, future developments in SM and SR optical microscopy techniques, and in the systems under study, have the potential to make even greater contributions to the HR field.

\section{Improving SR and SM technology}

SM and SR studies are technology-driven, and advances in these technologies offer the potential for greater spatial and temporal resolution, which in turn will lead to more detailed biophysical insights. For instance, faster cameras with greater spatial resolution and higher sensitivity and brighter, more stable fluorophores would enable higher-quality images to be collected over longer periods of time than is currently possible. These advances would also help overcome some of the technical problems that currently limit most SR studies to fixed samples, namely, slow image acquisition rates, challenges in labelling proteins in living cells and phototoxicity associated with the high laser illumination intensities ${ }^{113,114,118}$. Thus, SR imaging might eventually be used to look at HR-related reaction mechanisms in living cells as they are occurring in real time.

\section{Increasing the complexity of SM studies}

As with any reductionist biochemical approach, many of the SM studies of recombination have focused on understanding the detailed properties of one or two proteins in order to infer their particular contributions to the overall recombination pathway. Therefore, a major challenge faced in the SM field will be to gradually increase the complexity of the systems under investigation. For instance, most of the SM studies of recombinase filaments described above have been confined to analysis of only the recombinases (such as RecA or Rad51) and therefore reflect only the basal properties of these proteins ${ }^{23,98}$. However, it is clear from many years of genetic, cell biology and biochemical studies that the presynaptic complex contains many other proteins in addition to the recombinases, although the precise spatial organization of these proteins within the presynaptic complex remains ill-defined ${ }^{3,4,15,16,148}$. Moving forward, it will be essential to understand whether and how the presence of these additional recombination accessory proteins influences filament assembly and dynamics, the homology search and strand invasion. It will also be important to determine how these processes take place within the context of chromatin, how chromatin-remodelling factors work together with recombination machinery and how recombination is coordinated with DNA replication.

\section{Determining biological function with SR}

The structures that have been observed by SR imaging will require continued contributions from genetic, biochemical and biophysical studies to more fully define their mechanistic and biological implications. For instance, although SR studies of the synaptonemal complex have provided beautiful optical images of these structures and new insights into their organization, the impact of this organization on function has yet to be fully revealed ${ }^{133-140}$. While the potential of SR imaging is far from being fully realized, we anticipate continued and rapid developments in this powerful technology that will lead to broader applications ${ }^{114,118}$. For instance, traditional approaches have been unable to determine how chromosome organization contributes to homologue bias during meiotic recombination, but this problem may eventually prove amenable to SR imaging. 
In conclusion, as SM and SR studies continue to mature, they are likely to provide further insights into HR mechanisms. In the groundbreaking paper describing the DSBR model, the authors conclude that while genetic studies have been invaluable in the elucidation of recombination pathways, “...we suggest that biochemical experiments will be necessary to determine the actual mechanism of initiation of ... recombination" (REF. 5). We extend this sentiment by stating that the biochemical information must span a range of spatial and temporal scales and must also be integrated with an in-depth understanding of the biology of recombination and the consequences of its outcomes. The new optical microscopy tools available for SM and SR imaging of HR can help make this a reality, and the coming years should prove extremely fruitful for those investigating HR mechanisms.

\section{Acknowledgments}

The authors thank members of the Greene laboratory for their insights during the preparation of this manuscript. This research was funded by US National Institutes of Health grant R35GM118026 and by US National Science Foundation grant MCB-1154511 (E.C.G.). L.D.T. was supported by a Pew Latin American Fellowship, the Williams Foundation and Consejo Nacional de Investigaciones Científicas y Técnicas (CONICET), Argentina. The authors apologize to colleagues whose works were unable to be cited owing to length limitations.

\section{Glossary}

\section{Double-strand break repair}

(DSBR). An umbrella term that encompasses numerous potential pathways for the repair of a double-strand break. These pathways include non-homologous end joining, microhomology-mediated end joining, synthesis-dependent strand annealing and homologous recombination.

\section{Horizontal gene transfer}

The transfer of genetic material between the genomes of two organisms that does not occur through parent-progeny transmission. Also referred to as lateral gene transfer.

\section{Loss of heterozygosity}

A loss of one of the alleles at a given locus as a result of a genomic change, such as mitotic deletion, gene conversion or chromosome mis-segregation.

\section{Holliday model}

An early model of double-strand break repair that proposed a cross-stranded structure was the result of two single-stranded DNA breaks and accounted for both gene conversion and crossing over. While the model was later updated by Meselson and Radding, the crossover structure called the Holliday junction remains a cornerstone of recombination.

\section{Meselson-Radding model}

An update to the Holliday model that accounted for aberrant segregation in yeast tetrad analysis whereby recombination is initiated through a single double-stranded DNA break rather than two single-stranded DNA breaks. The model still employs the crossover structure first proposed by Holliday.

\section{Synthesis-dependent strand annealing}


(SDSA). A mode of double-strand break repair that proceeds through the early steps of homologous recombination but does not include second-end capture or the Holliday junction. Instead, after DNA synthesis primed by the initial captured end, the heteroduplex joint is dissociated to re-anneal with the second end of the double-strand break and serve as the template for further gap repair and resolution.

\section{Meiotic recombination}

A specialized type of homologous recombination that takes place during meiosis and is required to generate a physical linkage called a chiasma between two (non-sister) homologous chromosomes. Most, but not all, eukaryotes require the meiosis-specific recombinase meiotic recombination protein DMC1/LIM15 homologue (DMC1) to promote meiotic recombination.

\section{Single-molecule optical microscopy}

A class of techniques that use optical microscopy to study the biochemical and biophysical properties of biological molecules.

\section{Super-resolution optical microscopy}

A class of microscopy techniques used to enhance the spatial resolution of an optical microscope.

\section{RAD51/RecA family of DNA recombinases}

A highly conserved family of ATP-dependent DNA-binding proteins that promote critical steps in homologous recombination. Examples of key members of this family include bacteriophage $\mathrm{T} 4$ recombination and repair protein (UvsX), bacterial protein RecA, archaeal RadA, and the eukaryotic recombinases RAD51 and meiotic recombination protein DMC1/ LIM15 homologue (DMC1).

\section{Evanescent field}

An oscillating electromagnetic field that is spatially concentrated at the interface between two materials with different refractive indices, for instance, glass and water. Also known as an evanescent wave. In the case of total internal reflection microscopy, the evanescent field is confined near the interface between the aqueous buffer and the glass microscope slide (or coverslip).

\section{Single-molecule FRET}

(smFRET). A technique that allows the direct measurement of distances between macromolecules of interest within a range of $\sim 10-50 \AA$. Also referred to as single-pair FRET (spFRET). smFRET is commonly used with total internal reflection microscopy but can be adapted for use in many types of single-molecule imaging systems.

\section{Presynaptic complex}

A nucleoprotein complex that comprises the presynaptic single-stranded DNA and the associated protein cofactors necessary for promoting homologous recombination (HR). Key among these factors are the RAD51/RecA family of DNA recombinases, which act together with a number of associated factors to promote HR.

Nat Rev Genet. Author manuscript; available in PMC 2018 October 01. 


\section{Crossover hotspot instigator sites}

(chi sites). Specific cis-acting sites consisting of an eight-nucleotide DNA sequence ( $5^{\prime}$ GCTGGTGG-3') that is over-represented in the Escherichia coli genome and helps regulate the properties of the RecBCD complex by controlling the activities of the RecBCD enzyme subunits RecB and RecD ATP-dependent motor proteins.

\section{Translocation}

General term used to indicate the ATP-dependent movement of a motor protein (such as a helicase or a polymerase) along a nucleic acid.

\section{Processivity}

The tendency of a helicase (or any other nucleic acid motor protein) to continue to move along a nucleic acid molecule rather than dissociating into free solution.

\section{Nucleoprotein filaments}

The helical filament structures formed by members of the RAD51/RecA family of recombinases as they bind to single-stranded DNA. They contain one protein monomer for every three DNA bases and six protein monomers per helical turn. These nucleoprotein filaments are a key component of the presynaptic complex.

\section{Homology search}

The process during which the presynaptic complex searches the genome for a doublestranded DNA sequence that is homologous to the single-stranded DNA sequence present at the processed ends of a double-strand break.

\section{Strand invasion}

A reaction catalysed by the RAD51/RecA family of recombinases, which allows for Watson-Crick pairing interactions between a single-stranded DNA molecule and the complementary strand within a homologous double-stranded DNA, resulting in displacement of the non-complementary strand.

\section{D-Loop}

The paired intermediate generated by a successful strand invasion reaction wherein the $3^{\prime}$ end of the invading single-stranded DNA strand is now available as a primer for DNA synthesis.

\section{References}

1. Dunham MA, Neumann AA, Fasching CL, Reddel RR. Telomere maintenance by recombination in human cells. Nat Genet. 2000; 26:447-450. [PubMed: 11101843]

2. Jasin M, Rothstein R. Repair of strand breaks by homologous recombination. Cold Spring Harb Perspect Biol. 2013; 5:a012740. [PubMed: 24097900]

3. Symington LS, Rothstein R, Lisby M. Mechanisms and regulation of mitotic recombination in Saccharomyces cerevisiae. Genetics. 2014; 198:795-835. [PubMed: 25381364]

4. San Filippo J, Sung P, Klein H. Mechanism of eukaryotic homologous recombination. Annu Rev Biochem. 2008; 77:229-257. [PubMed: 18275380]

5. Szostak JW, Orr-Weaver TL, Rothstein RJ, Stahl FW. The double-strand-break repair model for recombination. Cell. 1983; 33:25-35. [PubMed: 6380756]

Nat Rev Genet. Author manuscript; available in PMC 2018 October 01. 
6. Chanet R, Heude M, Adjiri A, Maloisel L, Fabre F. Semidominant mutations in the yeast Rad51 protein and their relationships with the Srs2 helicase. Mol Cell Biol. 1996; 16:4782-4789. [PubMed: 8756636]

7. Kass EM, Moynahan ME, Jasin M. When genome maintenance goes badly awry. Mol Cell. 2016; 62:777-787. [PubMed: 27259208]

8. Moynahan ME, Jasin M. Mitotic homologous recombination maintains genomic stability and suppresses tumorigenesis. Nat Rev Mol Cell Biol. 2010; 11:196-207. [PubMed: 20177395]

9. Prakash R, Zhang Y, Feng W, Jasin M. Homologous recombination and human health: the roles of BRCA1, BRCA2, and associated proteins. Cold Spring Harb Perspect Biol. 2015; $7: a 016600$. [PubMed: 25833843]

10. Holliday R. A mechanism for gene conversion in fungi [Reprinted]. Genet Res. 2007; 89:285-307. [PubMed: 18976517]

11. Meselson MS, Radding CM. A general model for genetic recombination. Proc Natl Acad Sci USA. 1975; 72:358-361. [PubMed: 1054510]

12. Nassif N, Penney J, Pal S, Engels WR, Gloor GB. Efficient copying of nonhomologous sequences from ectopic sites via P-element-induced gap repair. Mol Cell Biol. 1994; 14:1613-1625. [PubMed: 8114699]

13. Mehta A, Haber JE. Sources of DNA double-strand breaks and models of recombinational DNA repair. Cold Spring Harb Perspect Biol. 2014; 6:a016428. [PubMed: 25104768]

14. Paques F, Haber JE. Multiple pathways of recombination induced by double-strand breaks in Saccharomyces cerevisiae. Microbiol Mol Biol Rev. 1999; 63:349-404. [PubMed: 10357855]

15. Kowalczykowski SC. An overview of the molecular mechanisms of recombinational DNA repair. Cold Spring Harbor Perspect Biol. 2015; 7:a016410.

16. Heyer WD, Ehmsen KT, Liu J. Regulation of homologous recombination in eukaryotes. Annu Rev Genet. 2010; 44:113-139. [PubMed: 20690856]

17. Morrical SW. DNA-pairing and annealing processes in homologous recombination and homologydirected repair. Cold Spring Harbor Perspect Biol. 2015; 7:a016444.

18. Prentiss M, Prevost C, Danilowicz C. Structure/function relationships in RecA protein-mediated homology recognition and strand exchange. Crit Rev Biochem Mol Biol. 2015; 50:453-476. [PubMed: 26459995]

19. Joo C, Balci H, Ishitsuka Y, Buranachai C, Ha T. Advances in single-molecule fluorescence methods for molecular biology. Annu Rev Biochem. 2008; 77:51-76. [PubMed: 18412538]

20. Monachino E, Spenkelink LM, van Oijen AM. Watching cellular machinery in action, one molecule at a time. J Cell Biol. 2017; 216:41-51. [PubMed: 27979907]

21. Roy R, Hohng S, Ha T. A practical guide to single-molecule FRET. Nat Methods. 2008; 5:507516. [PubMed: 18511918]

22. Walter NG, Huang CY, Manzo AJ, Sobhy MA. Do-it-yourself guide: how to use the modern single-molecule toolkit. Nat Methods. 2008; 5:475-489. [PubMed: 18511916]

23. Bell JC, Kowalczykowski SC. Mechanics and single-molecule interrogation of DNA recombination. Annu Rev Biochem. 2016; 85:193-226. [PubMed: 27088880]

24. van Mameren J, Peterman EJ, Wuite GJ. See me, feel me: methods to concurrently visualize and manipulate single DNA molecules and associated proteins. Nucleic Acids Res. 2008; 36:43814389. [PubMed: 18586820]

25. Axelrod D. Total internal reflection fluorescence microscopy. Methods Cell Biol. 1989; 30:245270. [PubMed: 2648112]

26. Reck-Peterson SL, Derr ND, Stuurman N. Imaging single molecules using total internal reflection fluorescence microscopy (TIRFM. Cold Spring Harb Protoc. 2010; 2010 pdb.top73.

27. Hoffman MT, Sheung J, Selvin PR. Fluorescence imaging with one nanometer accuracy: in vitro and in vivo studies of molecular motors. Methods Mol Biol. 2011; 778:33-56. [PubMed: 21809199]

28. Stryer L. Fluorescence energy transfer as a spectroscopic ruler. Annu Rev Biochem. 1978; 47:819846. [PubMed: 354506]

29. Lakowicz, J. Principles of Fluorescence Spectroscopy. 3. Springer; 2011. 
30. Ha T, et al. Single-molecule fluorescence spectroscopy of enzyme conformational dynamics and cleavage mechanism. Proc Natl Acad Sci USA. 1999; 96:893-898. [PubMed: 9927664]

31. Ma CJ, Steinfeld JB, Greene EC. Single-stranded DNA curtains for studying homologous recombination. Methods Enzymol. 2017; 582:193-219. [PubMed: 28062035]

32. Greene EC, Wind S, Fazio T, Gorman J, Visnapuu ML. DNA curtains for high-throughput singlemolecule optical imaging. Methods Enzymol. 2010; 472:293-315. [PubMed: 20580969]

33. Lohman TM, Tomko EJ, Wu CG. Nonhexameric DNA helicases and translocases: mechanisms and regulation. Nat Rev Mol Cell Biol. 2008; 9:391-401. [PubMed: 18414490]

34. Singleton MR, Dillingham MS, Wigley DB. Structure and mechanism of helicases and nucleic acid translocases. Annu Rev Biochem. 2007; 76:23-50. [PubMed: 17506634]

35. Pyle AM. Translocation and unwinding mechanisms of RNA and DNA helicases. Annu Rev Biophys. 2008; 37:317-336. [PubMed: 18573084]

36. Bernstein KA, Gangloff S, Rothstein R. The RecQ DNA helicases in DNA repair. Annu Rev Genet. 2010; 44:393-417. [PubMed: 21047263]

37. Brosh RM Jr. DNA helicases involved in DNA repair and their roles in cancer. Nat Rev Cancer. 2013; 13:542-558. [PubMed: 23842644]

38. Croteau DL, Popuri V, Opresko PL, Bohr VA. Human RecQ helicases in DNA repair, recombination, and replication. Annu Rev Biochem. 2014; 83:519-552. [PubMed: 24606147]

39. Niu H, Klein HL. Multifunctional roles of Saccharomyces cerevisiae Srs2 protein in replication, recombination and repair. FEMS Yeast Res. 2017; 17:fow11.

40. Vannier JB, Sarek G, Boulton SJ. RTEL1: functions of a disease-associated helicase. Trends Cell Biol. 2014; 24:416-425. [PubMed: 24582487]

41. Branzei D, Foiani M. RecQ helicases queuing with Srs2 to disrupt Rad51 filaments and suppress recombination. Genes Dev. 2007; 21:3019-3026. [PubMed: 18056418]

42. Branzei D, Szakal B. Building up and breaking down: mechanisms controlling recombination during replication. Crit Rev Biochem Mol Biol. 2017; 52:381-394. [PubMed: 28325102]

43. Marini V, Krejci L. Srs2: the “Odd-Job Man” in DNA repair. DNA Repair. 2010; 9:268-275. [PubMed: 20096651]

44. Symington LS, Heyer WD. Some disassembly required: role of DNA translocases in the disruption of recombination intermediates and dead-end complexes. Genes Dev. 2006; 20:2479-2486. [PubMed: 16980577]

45. Dillingham MS, Kowalczykowski SC. RecBCD enzyme and the repair of double-stranded DNA breaks. Microbiol Mol Biol Rev. 2008; 72:642-671. [PubMed: 19052323]

46. Smith GR. How RecBCD enzyme and Chi promote DNA break repair and recombination: a molecular biologist's view. Microbiol Mol Biol Rev. 2012; 76:217-228. [PubMed: 22688812]

47. Wigley DB. Bacterial DNA repair: recent insights into the mechanism of RecBCD, AddAB and AdnAB. Nat Rev Microbiol. 2013; 11:9-13. [PubMed: 23202527]

48. Bianco PR, et al. Processive translocation and DNA unwinding by individual RecBCD enzyme molecules. Nature. 2001; 409:374-378. This paper presents the earliest example of the application of SM methods to the DNA-end-processing molecular motor complex RecBCD. [PubMed: 11201750]

49. Spies M, et al. A molecular throttle: the recombination hotspot chi controls DNA translocation by the RecBCD helicase. Cell. 2003; 114:647-654. This paper describes the first SM study to demonstrate that chi recognition causes a change in the velocity of RecBCD that corresponds to a switch in the RecD and RecB motor activities. [PubMed: 13678587]

50. Ha T, et al. Initiation and re-initiation of DNA unwinding by the Escherichia coli Rep helicase. Nature. 2002; 419:638-641. [PubMed: 12374984]

51. Myong S, Rasnik I, Joo C, Lohman TM, Ha T. Repetitive shuttling of a motor protein on DNA. Nature. 2005; 437:1321-1325. [PubMed: 16251956]

52. Qiu Y, et al. Srs2 prevents Rad51 filament formation by repetitive motion on DNA. Nat Commun. 2013; 4:2281. [PubMed: 23939144]

53. Krejci L, et al. DNA helicase Srs2 disrupts the Rad51 presynaptic filament. Nature. 2003; 423:305-309. [PubMed: 12748644] 
54. Veaute X, et al. The Srs 2 helicase prevents recombination by disrupting Rad51 nucleoprotein filaments. Nature. 2003; 423:309-312. [PubMed: 12748645]

55. Park J, et al. PcrA helicase dismantles RecA filaments by reeling in DNA in uniform steps. Cell. 2010; 142:544-555. This paper describes the use of smFRET experiments to study how the helicase PcrA removes RecA from ssDNA. [PubMed: 20723756]

56. Petrova V, et al. Active displacement of RecA filaments by UvrD translocase activity. Nucleic Acids Res. 2015; 43:4133-4149. [PubMed: 25824953]

57. Anand SP, Zheng H, Bianco PR, Leuba SH, Khan SA. DNA helicase activity of PcrA is not required for the displacement of RecA protein from DNA or inhibition of RecA-mediated strand exchange. J Bacteriol. 2007; 189:4502-4509. [PubMed: 17449621]

58. Fagerburg MV, et al. PcrA-mediated disruption of RecA nucleoprotein filaments - essential role of the ATPase activity of RecA. Nucleic Acids Res. 2012; 40:8416-8424. [PubMed: 22743269]

59. Arslan S, Khafizov R, Thomas CD, Chemla YR, Ha T. Engineering of a superhelicase through conformational control. Science. 2015; 348:344-347. In this study, researchers engineer Rep and PcrA 'superhelicases' that are 'locked' into highly active conformational states and use them to define the relationship between their structures and their functions. [PubMed: 25883358]

60. Comstock MJ, et al. Direct observation of structure-function relationship in a nucleic acidprocessing enzyme. Science. 2015; 348:352-354. In this study, researchers use smFRET in conjunction with DNA force measurements to directly visualize the helicase UvrD acting on DNA. The findings indicate how protein conformational changes and changes in oligomeric state can influence helicase activities. [PubMed: 25883359]

61. Korolev S, Hsieh J, Gauss GH, Lohman TM, Waksman G. Major domain swiveling revealed by the crystal structures of complexes of $E$ coli Rep helicase bound to single-stranded DNA and ADP. Cell. 1997; 90:635-647. [PubMed: 9288744]

62. Lee JY, Yang W. UvrD helicase unwinds DNA one base pair at a time by a two-part power stroke. Cell. 2006; 127:1349-1360. [PubMed: 17190599]

63. Velankar SS, Soultanas P, Dillingham MS, Subramanya HS, Wigley DB. Crystal structures of complexes of PcrA DNA helicase with a DNA substrate indicate an inchworm mechanism. Cell. 1999; 97:75-84. [PubMed: 10199404]

64. Wold MS. Replication protein A: a heterotrimeric, single-stranded DNA-binding protein required for eukaryotic DNA metabolism. Annu Rev Biochem. 1997; 66:61-92. [PubMed: 9242902]

65. Meyer RR, Laine PS. The single-stranded DNA-binding protein of Escherichia coli. Microbiol Rev. 1990; 54:342-380. [PubMed: 2087220]

66. Bianco PR. The tale of SSB. Progress Biophys Mol Biol. 2017; 127:111-118.

67. Chen R, Wold MS. Replication protein A: single-stranded DNA's first responder: dynamic DNAinteractions allow replication protein A to direct single-strand DNA intermediates into different pathways for synthesis or repair. BioEssays. 2014; 36:1156-1161. [PubMed: 25171654]

68. Fanning E, Klimovich V, Nager AR. A dynamic model for replication protein A (RPA) function in DNA processing pathways. Nucleic Acids Res. 2006; 34:4126-4137. [PubMed: 16935876]

69. Krejci L, Sung P. RPA not that different from SSB. Structure. 2002; 10:601-602. [PubMed: 12015141]

70. Shereda RD, Kozlov AG, Lohman TM, Cox MM, Keck JL. SSB as an organizer/mobilizer of genome maintenance complexes. Crit Rev Biochem Mol Biol. 2008; 43:289-318. [PubMed: 18937104]

71. Marechal A, Zou L. RPA-coated single-stranded DNA as a platform for post-translational modifications in the DNA damage response. Cell Res. 2015; 25:9-23. [PubMed: 25403473]

72. Gibb B, Silverstein TD, Finkelstein IJ, Greene EC. Single-stranded DNA curtains for real-time single-molecule visualization of protein-nucleic acid interactions. Anal Chem. 2012; 84:76077612. [PubMed: 22950646]

73. Gibb B, et al. Concentration-dependent exchange of replication protein A on single-stranded DNA revealed by single-molecule imaging. PLoS ONE. 2014; 9:e87922. [PubMed: 24498402]

74. Deng SK, Gibb B, de Almeida MJ, Greene EC, Symington LS. RPA antagonizes microhomologymediated repair of DNA double-strand breaks. Nat Struct Mol Biol. 2014; 21:405-412. [PubMed: 24608368] 
75. Ma CJ, Gibb B, Kwon Y, Sung P, Greene EC. Protein dynamics of human RPA and RAD51 on ssDNA during assembly and disassembly of the RAD51 filament. Nucleic Acids Res. 2017; 45:749-761. [PubMed: 27903895]

76. Graham JS, Johnson RC, Marko JF. Concentration-dependent exchange accelerates turnover of proteins bound to double-stranded DNA. Nucleic Acids Res. 2011; 39:2249-2259. In this paper, SM imaging is used to show that DNA-binding proteins Fis and $\mathrm{HU}$ and non-histone chromosomal protein 6A (Nhp6A) can undergo concentration-dependent exchange between free and bound states, thus defining a new mechanism that can contribute to protein-nucleic acid interaction dynamics. [PubMed: 21097894]

77. Chen TY, et al. Concentration- and chromosome-organization- dependent regulator unbinding from DNA for transcription regulation in living cells. Nat Commun. 2015; 6:7445. [PubMed: 26145755]

78. Ha T. Single-molecule approaches embrace molecular cohorts. Cell. 2013; 154:723-726. [PubMed: 23953107]

79. Aberg C, Duderstadt KE, van Oijen AM. Stability versus exchange: a paradox in DNA replication. Nucleic Acids Res. 2016; 44:4846-4854. [PubMed: 27112565]

80. Roy R, Kozlov AG, Lohman TM, Ha T. SSB protein diffusion on single-stranded DNA stimulates RecA filament formation. Nature. 2009; 461:1092-1097. [PubMed: 19820696]

81. Sokoloski JE, Kozlov AG, Galletto R, Lohman TM. Chemo-mechanical pushing of proteins along single-stranded DNA. Proc Natl Acad Sci USA. 2016; 113:6194-6199. [PubMed: 27185951]

82. Bell JC, Liu B, Kowalczykowski SC. Imaging and energetics of single SSB-ssDNA molecules reveal intramolecular condensation and insight into RecOR function. eLife. 2015; 4:e08646. [PubMed: 26381353]

83. Myler LR, et al. Single-molecule imaging reveals the mechanism of Exo1 regulation by singlestranded DNA binding proteins. Proc Natl Acad Sci USA. 2016; 113:E1170-E1179. [PubMed: 26884156]

84. Bianco PR, Tracy RB, Kowalczykowski SC. DNA strand exchange proteins: a biochemical and physical comparison. Front Biosci. 1998; 3:D570-D603. [PubMed: 9632377]

85. Joo C, et al. Real-time observation of RecA filament dynamics with single monomer resolution. Cell. 2006; 126:515-527. [PubMed: 16901785]

86. Bell JC, Kowalczykowski SC. RecA: regulation and mechanism of a molecular search engine. Trends Biochem Sci. 2016; 41:491-507. [PubMed: 27156117]

87. Galletto R, Amitani I, Baskin RJ, Kowalczykowski SC. Direct observation of individual RecA filaments assembling on single DNA molecules. Nature. 2006; 443:875-878. In this study, researchers use a multichannel flow chamber system to directly visualize the assembly of RecA filaments on single dsDNA molecules, providing new insight into filament nucleation and filament growth. [PubMed: 16988658]

88. Bell JC, Plank JL, Dombrowski CC, Kowalczykowski SC. Direct imaging of RecA nucleation and growth on single molecules of SSB-coated ssDNA. Nature. 2012; 491:274-278. In this work, new insights into the presynaptic complex assembly mechanism are generated by using multicolour SM imaging to study the assembly of RecA filaments on single molecules of SSB-bound ssDNA in the presence or absence of the RecFOR proteins. [PubMed: 23103864]

89. Morimatsu K, Kowalczykowski SC. RecFOR proteins load RecA protein onto gapped DNA to accelerate DNA strand exchange: a universal step of recombinational repair. Mol Cell. 2003; 11:1337-1347. [PubMed: 12769856]

90. Umezu K, Chi NW, Kolodner RD. Biochemical interaction of the Escherichia coli RecF, RecO, and RecR proteins with RecA protein and single-stranded DNA binding protein. Proc Natl Acad Sci USA. 1993; 90:3875-3879. [PubMed: 8483906]

91. Gibb B, et al. Protein dynamics during presynaptic-complex assembly on individual singlestranded DNA molecules. Nat Struct Mol Biol. 2014; 21:893-900. [PubMed: 25195049]

92. Lee JY, Qi Z, Greene EC. ATP hydrolysis promotes duplex DNA release by the RecA presynaptic complex. J Biol Chem. 2016; 291:22218-22230. [PubMed: 27587394]

93. Lee JY, et al. Base triplet stepping by the Rad51/RecA family of recombinases. Science. 2015; 349:977-981. [PubMed: 26315438] 
94. Qi Z, et al. DNA sequence alignment by microhomology sampling during homologous recombination. Cell. 2015; 160:856-869. [PubMed: 25684365]

95. Taylor MR, et al. A polar and nucleotide-dependent mechanism of action for RAD51 paralogs in RAD51 filament remodeling. Mol Cell. 2016; 64:926-939. [PubMed: 27867009]

96. Ogawa T, Yu X, Shinohara A, Egelman EH. Similarity of the yeast RAD51 filament to the bacterial RecA filament. Science. 1993; 259:1896-1899. [PubMed: 8456314]

97. van Mameren J, et al. Counting RAD51 proteins disassembling from nucleoprotein filaments under tension. Nature. 2009; 457:745-748. [PubMed: 19060884]

98. Candelli A, Modesti M, Peterman EJ, Wuite GJ. Single-molecule views on homologous recombination. Q Rev Biophys. 2013; 46:323-348. [PubMed: 24016421]

99. Candelli A, Wuite GJ, Peterman EJ. Combining optical trapping, fluorescence microscopy and micro-fluidics for single molecule studies of DNA-protein interactions. Phys Chem Chem Phys. 2011; 13:7263-7272. [PubMed: 21416086]

100. Mameren J, et al. Dissecting elastic heterogeneity along DNA molecules coated partly with Rad51 using concurrent fluorescence microscopy and optical tweezers. Biophys J. 2006; 91:L78L80. [PubMed: 16920830]

101. Greene EC. DNA sequence alignment during homologous recombination. J Biol Chem. 2016; 291:11572-11580. [PubMed: 27129270]

102. Renkawitz J, Lademann CA, Jentsch S. Mechanisms and principles of homology search during recombination. Nat Rev Mol Cell Biol. 2014; 15:369-383. [PubMed: 24824069]

103. Barzel A, Kupiec M. Finding a match: how do homologous sequences get together for recombination? Nat Rev Genet. 2008; 9:27-37. [PubMed: 18040271]

104. Gonda DK, Radding CM. The mechanism of the search for homology promoted by recA protein. Facilitated diffusion within nucleoprotein networks. J Biol Chem. 1986; 261:13087-13096. [PubMed: 3020024]

105. Forget AL, Kowalczykowski SC. Single-molecule imaging of DNA pairing by RecA reveals a three-dimensional homology search. Nature. 2012; 482:423-427. In this study, a dual optical trap is used to manipulate the end-to-end length of a single dsDNA molecule while concurrently visualizing single RecA presynaptic complexes as they search the dsDNA for homology. This work demonstrates that the homology search is a 3-dimensional process, which involves multivalent contacts between the presynaptic complex and the dsDNA. [PubMed: 22318518]

106. Ragunathan K, Liu C, Ha T. RecA filament sliding on DNA facilitates homology search. eLife. 2012; 1:e00067. [PubMed: 23240082]

107. De Vlaminck I, et al. Mechanism of homology recognition in DNA recombination from dualmolecule experiments. Mol Cell. 2012; 46:616-624. [PubMed: 22560720]

108. Jiang L, Prentiss M. RecA-mediated sequence homology recognition as an example of how searching speed in self-assembly systems can be optimized by balancing entropic and enthalpic barriers. Phys Rev E Stat Nonlin Soft Matter Phys. 2014; 90:022704. [PubMed: 25215755]

109. Kates-Harbeck J, Tilloy A, Prentiss M. Simplified biased random walk model for RecA-proteinmediated homology recognition offers rapid and accurate self-assembly of long linear arrays of binding sites. Phys Rev E Stat Nonlin Soft Matter Phys. 2013; 88:012702. [PubMed: 23944487]

110. Thomas CA Jr. Recombination of DNA molecules. Prog Nucleic Acid Res Mol Biol. 1966; 5:315-337. [PubMed: 5337698]

111. Danilowicz C, et al. RecA homology search is promoted by mechanical stress along the scanned duplex DNA. Nucleic Acids Res. 2012; 40:1717-1727. [PubMed: 22013164]

112. Ragunathan K, Joo C, Ha T. Real-time observation of strand exchange reaction with high spatiotemporal resolution. Structure. 2011; 19:1064-1073. [PubMed: 21827943]

113. Sahl SJ, Hell SW, Jakobs S. Fluorescence nanoscopy in cell biology. Nat Rev Mol Cell Biol. 2017; 16:685-701.

114. Liu Z, Lavis LD, Betzig E. Imaging live-cell dynamics and structure at the single-molecule level. Mol Cell. 2015; 58:644-659. [PubMed: 26000849]

115. Schermelleh L, Heintzmann R, Leonhardt H. A guide to super-resolution fluorescence microscopy. J Cell Biol. 2010; 190:165-175. [PubMed: 20643879]

Nat Rev Genet. Author manuscript; available in PMC 2018 October 01. 
116. Thompson MA, Lew MD, Moerner WE. Extending microscopic resolution with single-molecule imaging and active control. Annu Rev Biophys. 2012; 41:321-342. [PubMed: 22577822]

117. Sengupta P, van Engelenburg SB, Lippincott-Schwartz J. Superresolution imaging of biological systems using photoactivated localization microscopy. Chem Rev. 2014; 114:3189-3202. [PubMed: 24417572]

118. Lambert TJ, Waters JC. Navigating challenges in the application of superresolution microscopy. J Cell Biol. 2017; 216:53-63. [PubMed: 27920217]

119. Betzig E. Single molecules, cells, and super-resolution optics (Nobel Lecture). Angewandte Chemie (Int Ed). 2015; 54:8034-8053.

120. Bates M, Huang B, Dempsey GT, Zhuang X. Multicolor super-resolution imaging with photoswitchable fluorescent probes. Science. 2007; 317:1749-1753. [PubMed: 17702910]

121. Huang B, Wang W, Bates M, Zhuang X. Three-dimensional super-resolution imaging by stochastic optical reconstruction microscopy. Science. 2008; 319:810-813. [PubMed: 18174397]

122. Rust MJ, Bates M, Zhuang X. Sub-diffraction-limit imaging by stochastic optical reconstruction microscopy (STORM). Nat Methods. 2006; 3:793-795. [PubMed: 16896339]

123. Betzig E, et al. Imaging intracellular fluorescent proteins at nanometer resolution. Science. 2006; 313:1642-1645. [PubMed: 16902090]

124. Manley S, et al. High-density mapping of single-molecule trajectories with photoactivated localization microscopy. Nat Methods. 2008; 5:155-157. [PubMed: 18193054]

125. Sengupta P, Van Engelenburg S, Lippincott-Schwartz J. Visualizing cell structure and function with point-localization superresolution imaging. Dev Cell. 2012; 23:1092-1102. [PubMed: 23237943]

126. Hell SW, Wichmann J. Breaking the diffraction resolution limit by stimulated emission: stimulate-demission-depletion fluorescence microscopy. Opt Lett. 1994; 19:780-782. [PubMed: 19844443]

127. Klar TA, Hell SW. Subdiffraction resolution in far-field fluorescence microscopy. Opt Lett. 1999; 24:954-956. [PubMed: 18073907]

128. Westphal V, et al. Video-rate far-field optical nanoscopy dissects synaptic vesicle movement. Science. 2008; 320:246-249. [PubMed: 18292304]

129. Gustafsson MG. Surpassing the lateral resolution limit by a factor of two using structured illumination microscopy. J Microsc. 2000; 198:82-87. [PubMed: 10810003]

130. Gustafsson MG. Nonlinear structured-illumination microscopy: wide-field fluorescence imaging with theoretically unlimited resolution. Proc Natl Acad Sci USA. 2005; 102:13081-13086. [PubMed: 16141335]

131. Schermelleh L, et al. Subdiffraction multicolor imaging of the nuclear periphery with $3 \mathrm{D}$ structured illumination microscopy. Science. 2008; 320:1332-1336. [PubMed: 18535242]

132. Neale MJ, Keeney S. Clarifying the mechanics of DNA strand exchange in meiotic recombination. Nature. 2006; 442:153-158. [PubMed: 16838012]

133. Keeney S, Lange J, Mohibullah N. Self-organization of meiotic recombination initiation: general principles and molecular pathways. Annu Rev Genet. 2014; 48:187-214. [PubMed: 25421598]

134. Hunter N. Meiotic recombination: the essence of heredity. Cold Spring Harb Perspect Biol. 2015; 7:a016618. [PubMed: 26511629]

135. Zickler D, Kleckner N. Recombination, pairing, and synapsis of homologs during meiosis. Cold Spring Harb Perspect Biol. 2015; 7:a016626. [PubMed: 25986558]

136. Zickler D, Kleckner N. Meiotic chromosomes: integrating structure and function. Annu Rev Genet. 1999; 33:603-754. [PubMed: 10690419]

137. Schucker K, Holm T, Franke C, Sauer M, Benavente R. Elucidation of synaptonemal complex organization by super-resolution imaging with isotropic resolution. Proc Natl Acad Sci USA. 2015; 112:2029-2033. [PubMed: 25646409]

138. Prakash K, et al. Superresolution imaging reveals structurally distinct periodic patterns of chromatin along pachytene chromosomes. Proc Natl Acad Sci USA. 2015; 112:14635-14640. [PubMed: 26561583] 
139. Cahoon CK, et al. Superresolution expansion microscopy reveals the three-dimensional organization of the Drosophila synaptonemal complex. Proc Natl Acad Sci USA. 2017; 114:E6857-E6866. In this study, the 3-dimensional structure of the D. melanogaster synaptonemal complex is examined using SIM. The resulting images indicate that the synaptonemal complex is organized into two distinct layers that might represent separation of the sister chromatids in 3D space. [PubMed: 28760978]

140. Kohler S, Wojcik M, Xu K, Dernburg AF. Superresolution microscopy reveals the threedimensional organization of meiotic chromosome axes in intact Caenorhabditis elegans tissue. Proc Natl Acad Sci USA. 2017; 114:E4734-E4743. In this study, STORM and PALM are used to image protein components of the $C$. elegans synaptonemal complex, which provides a detailed map of the 3D positions of these proteins and also suggests how they might help to compartmentalize sister chromatids. [PubMed: 28559338]

141. Brown MS, Bishop DK. DNA strand exchange and RecA homologs in meiosis. Cold Spring Harb Perspect Biol. 2014; 7:a016659. [PubMed: 25475089]

142. Bishop DK. RecA homologs Dmc1 and Rad51 interact to form multiple nuclear complexes prior to meiotic chromosome synapsis. Cell. 1994; 79:1081-1092. [PubMed: 7528104]

143. Shinohara M, Gasior SL, Bishop DK, Shinohara A. Tid1/Rdh54 promotes colocalization of rad51 and dmc1 during meiotic recombination. Proc Natl Acad Sci USA. 2000; 97:10814-10819. [PubMed: 11005857]

144. Kurzbauer MT, Uanschou C, Chen D, Schlogelhofer P. The recombinases DMC1 and RAD51 are functionally and spatially separated during meiosis in Arabidopsis. Plant Cell. 2012; 24:20582070. [PubMed: 22589466]

145. Bishop DK, et al. High copy number suppression of the meiotic arrest caused by a dmc1 mutation: REC114 imposes an early recombination block and RAD54 promotes a DMC1independent DSB repair pathway. Genes Cells. 1999; 4:425-444. [PubMed: 10526232]

146. Brown MS, Grubb J, Zhang A, Rust MJ, Bishop DK. Small Rad51 and Dmc1 complexes often co-occupy both ends of a meiotic DNA double strand break. PLoS Genet. 2015; 11:e1005653. In this study, STORM imaging is used to probe the properties of presynaptic complexes during meiosis and provides direct evidence that RAD51 and DMC1 co-occupy DNA ends and that these complexes are much smaller than had been previously anticipated. [PubMed: 26719980]

147. Lesterlin C, Ball G, Schermelleh L, Sherratt DJ. RecA bundles mediate homology pairing between distant sisters during DNA break repair. Nature. 2014; 506:249-253. This study provides an important example of live-cell SIM being used to examine the properties of a recombination protein-DNA complex in E. coli, which provides new insights into how RecA might act while trying to align DNA sequences in vivo. [PubMed: 24362571]

148. Lisby M, Rothstein R. Cell biology of mitotic recombination. Cold Spring Harb Perspect Biol. 2015; 7:a016535. [PubMed: 25731763]

149. Reindl J, et al. Chromatin organization revealed by nanostructure of irradiation induced $\gamma \mathrm{H} 2 \mathrm{AX}$, 53BP1 and Rad51 foci. Sci Rep. 2017; 7:40616. [PubMed: 28094292]

150. Liu B, Baskin RJ, Kowalczykowski SC. DNA unwinding heterogeneity by RecBCD results from static molecules able to equilibrate. Nature. 2013; 500:482-485. [PubMed: 23851395]

Nat Rev Genet. Author manuscript; available in PMC 2018 October 01. 


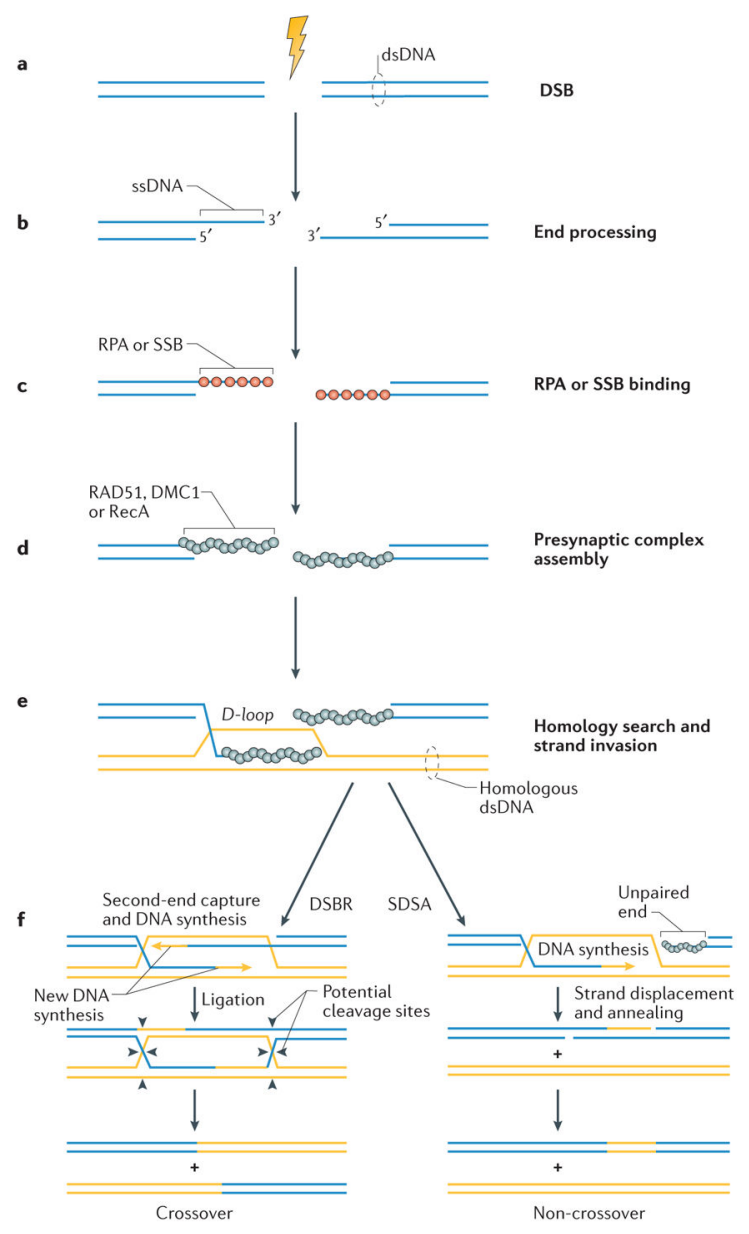

Figure 1. Overview of homologous recombination

a | The canonical homologous recombination pathway begins with formation of a doublestrand break (DSB). b | DSB formation is followed by end-processing reactions in which the $5^{\prime}$ single-stranded DNA (ssDNA) ends that flank DSBs are resected to expose 3' ssDNA ends. $\mathbf{c}$ These ends are quickly bound by ssDNA-binding proteins - single-stranded DNAbinding protein (SSB) in bacteria or replication protein A (RPA) in eukaryotes - that protect the ssDNA from degradation and remove any secondary structure. $\mathbf{d} \mid$ Mediator proteins (such as the Escherichia coli RecFOR proteins, Saccharomyces cerevisiae DNA repair and recombination protein Rad52 or human breast cancer type 2 susceptibility protein (BRCA2); not depicted) then stimulate the replacement of ssDNA-binding proteins with recombinases from the highly conserved RAD51/RecA family of DNA recombinases, which are all ATP-dependent DNA-binding proteins that form extended helical filaments on DNA. These filaments are referred to as presynaptic complexes, and they catalyse all the key DNA transactions that occur during the early stages of recombination. $\mathbf{e}$ The presynaptic filament first performs a homology search to locate a donor double-stranded DNA (dsDNA) molecule with sequence homology to the presynaptic ssDNA. Once a homologous donor has been identified, the presynaptic complex catalyses strand invasion, which results in the formation of a D-loop intermediate whereby the presynaptic ssDNA is paired with the homologous donor DNA. f $\mid$ The $3^{\prime}$ end of the invading ssDNA strand within the D-loop then serves as a 
primer for DNA polymerases to begin copying information from the donor template to complete repair of the damaged DNA through either the double-strand break repair (DSBR) pathway or the synthesis-dependent strand annealing (SDSA) pathway. During DSBR, both of the $3^{\prime}$ overhangs from the processed DSB are paired with the donor DNA, and subsequent ligation of the newly replicated ends results in the formation of a double Holliday junction. Resolution of the double Holliday junction by cleavage of the DNA strands can yield either crossover products (as depicted) or non-crossover products (not shown). During SDSA, only one of the $3^{\prime}$ overhangs is paired with the donor DNA, and the second end is thought to remain unpaired. The $3^{\prime}$ invading end is used as a primer for DNA replication. The newly synthesized strand is then displaced from the donor DNA and annealed to the complementary $3^{\prime}$ overhang from the other end of the processed DSB. Flap removal and ligation restores the broken DNA, producing only non-crossover products. We refer readers to several reviews for more detailed descriptions of each of these reaction steps $^{3,4,14,15,17}$. DMC1, meiotic recombination protein DMC1/LIM15 homologue. 
a
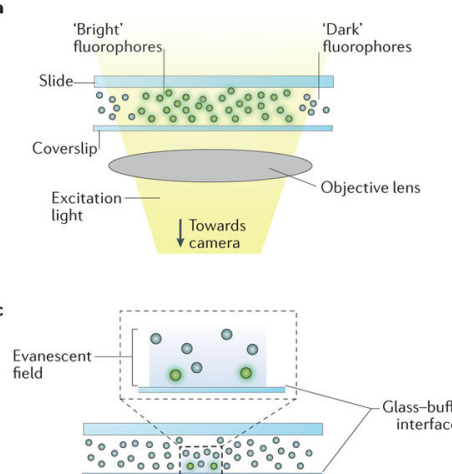

Optical trap
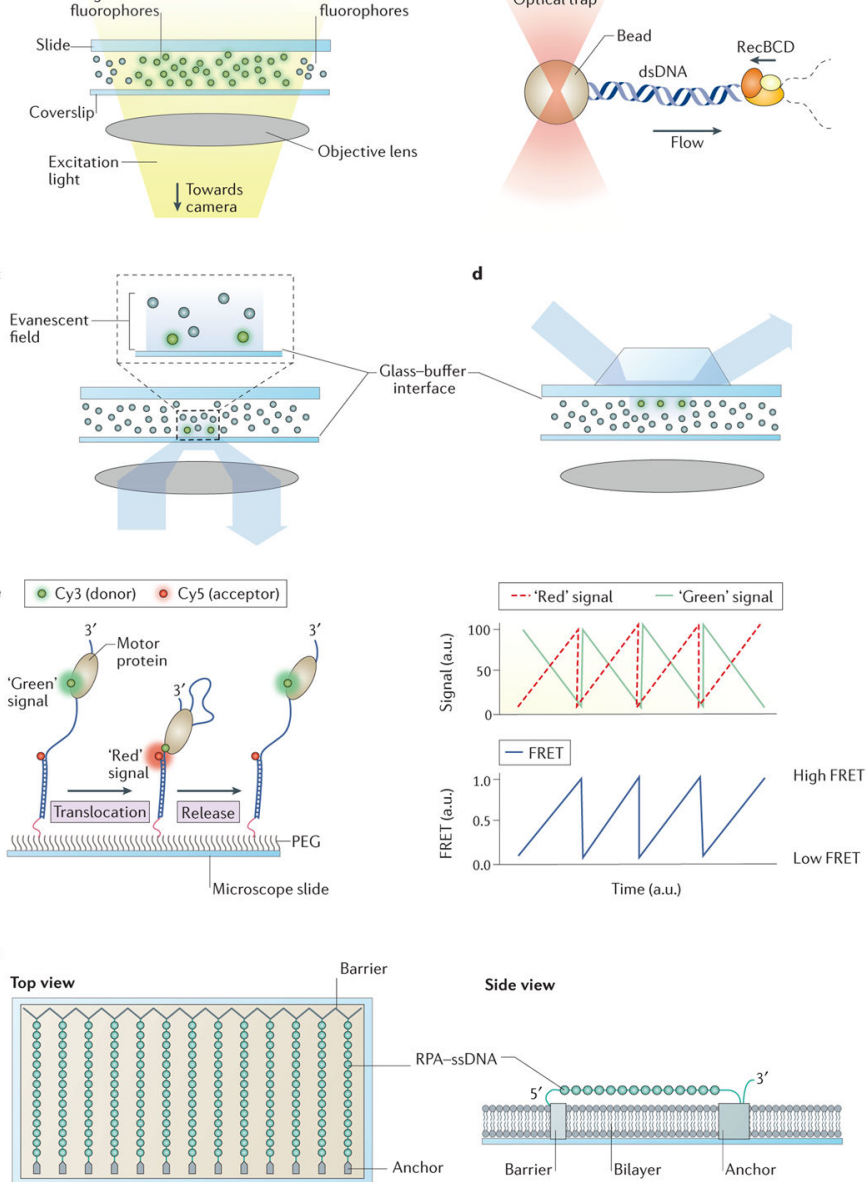

Side view

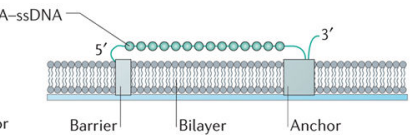

Figure 2. Examples of single-molecule imaging methods

a Simplified illustration of the illumination geometry used for typical epifluorescence microscopy. The excitation light passes through the entire sample volume, so all fluorophores present within this volume will contribute to the signal detected by the camera. Therefore, with this method, it is essential to minimize the concentration of free fluorescently tagged molecules present in the sample. $\mathbf{b} \mid$ Schematic illustration of an optical trapping setup that is often used in conjunction with epifluorescence microscopy. In this case, an infrared laser (shown in red) is used to trap a polystyrene bead. The bead is attached to one end of a fluorescently labelled DNA molecule (shown in blue), typically through a biotin- streptavidin linkage, and the trap can be used to position the bead in the sample chamber for optimal viewing. In the illustration, RecBCD is bound to the end of the DNA, and RecBCD helicase and/or nuclease activity would be revealed as a reduction in the length of the double-stranded DNA (dsDNA) in real time. $\mathbf{c}$ | Illumination geometry used for typical objective-type total internal reflection microscopy (TIRFM). Here, a laser beam is passed through the objective lens and reflected off the interface between the coverslip and the aqueous buffer holding the sample. This generates an evanescent field, which penetrates $\sim 100-300 \mathrm{~nm}$ into the sample chamber. The key feature of TIRFM is that the only fluorophores that will emit photons are those that are within the evanescent field. $\mathbf{d}$ | Schematic showing the illumination geometry for prism-type TIRFM. In this case, the laser 
is directed through a prism placed on top of the microscope slide glass. e $\mid$ Schematic illustration of how single-molecule fluorescence resonance energy transfer (smFRET) works and how it can be applied to studying protein movement on DNA. Here, a hypothetical motor protein is labelled with a 'green' fluorescent donor dye (Cy3), and the dsDNA-singlestranded DNA (ssDNA) junction is labelled with a 'red' fluorescent acceptor dye (Cy5). The green signal is excited with a laser of an appropriate wavelength, and only a green signal is emitted when the protein is far away from the junction (that is, $>50 \AA$ ). The red signal becomes visible as the protein approaches the junction because FRET occurs between the green donor dye and red acceptor dye, causing the red dye to emit photons. The resulting data can be expressed in graphical form as separate red and green signals, which should be anti-correlated, or as a FRET value, which is essentially a ratio of the two signals. Although compatible with other types of fluorescence microscopy, smFRET is often used with TIRFM, which usually requires that the molecules of interest be tethered to a surface to confine them to the evanescent field. In the example shown, the DNA is attached to a microscope slide through a biotin-streptavidin linkage, and the surface is covalently modified with polyethylene glycol (PEG) to prevent proteins or DNA from adhering to the glass. f | Schematic illustration of DNA curtains. Here, the individual strands of DNA are anchored at one end to a lipid bilayer through a biotin-streptavidin linkage and then aligned along the leading edge of a nanofabricated chromium barrier by application of buffer flow. The other end of the DNA can be attached nonspecifically to a series of anchor points so that the molecules are confined within the evanescent field of a total internal reflection microscope. In the example shown, ssDNA is coated with fluorescently labelled replication protein A (RPA) (a GFP- RPA fusion protein) (in green), which allows visualization of the DNA. Part $\mathbf{b}$ is adapted from REF. 150, Macmillan Publishers Limited. a.u., arbitrary units. 

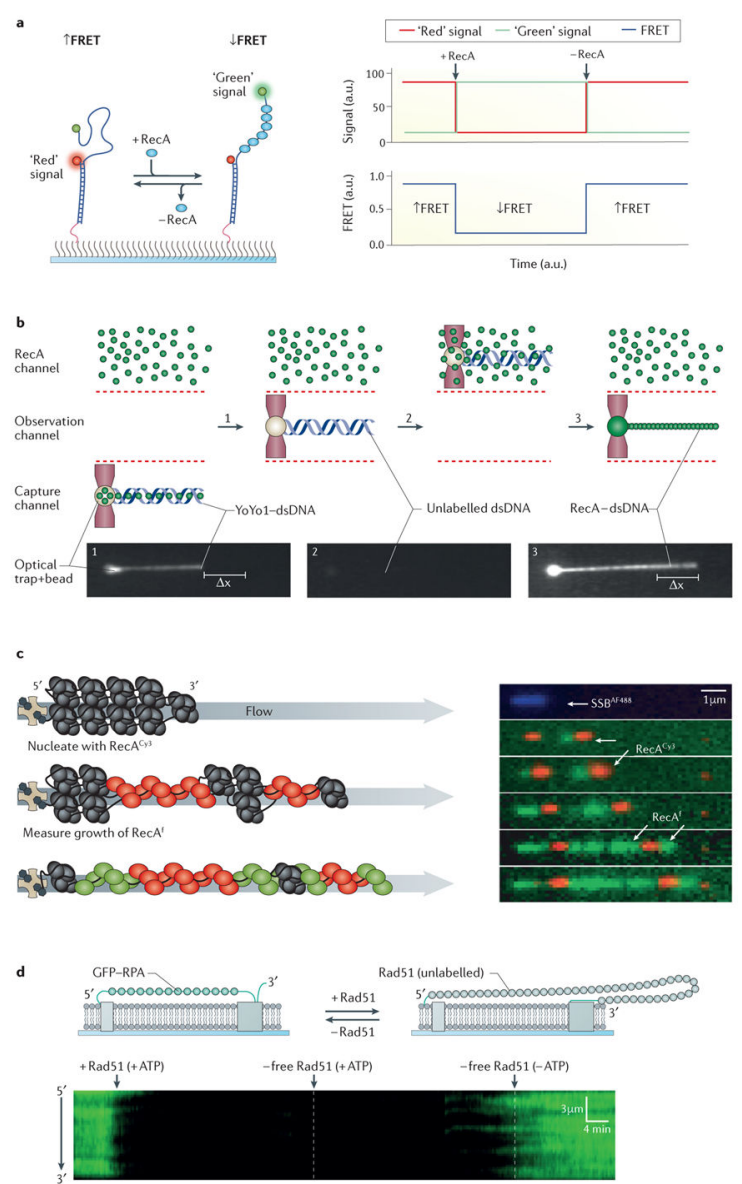

Figure 3. Examples of single-molecule experiments

a Single-molecule fluorescence resonance energy transfer (smFRET) assay for monitoring protein RecA binding to a short single-stranded DNA (ssDNA) overhang ${ }^{85}$. Left panel, formation of the RecA filament extends and stiffens the ssDNA, resulting in a low FRET state as the donor and acceptor dyes move away from one another. Right panel, schematic depiction of signal traces illustrating how the addition or removal of RecA results in changes in the 'red' and 'green' fluorescent signals and corresponding changes in SmFRET. b | Three-channel system for monitoring the DNA-binding activity of fluorescein-labelled RecA by epifluorescence microscopy ${ }^{87}$. Bead-tethered double-stranded DNA (dsDNA) is labelled with the intercalating dye YoYol and then captured in an optical trap. The DNA is then moved to the observation channel, which lacks free YoYo1 and contains $\operatorname{Mg}(\mathrm{OAc})^{2}$, which causes YoYo1 dissociation. The DNA is then 'dipped' into the RecA channel, but the DNA cannot be imaged in this channel because of the high background signal from the free RecA. The DNA is then brought back into the observation channel for imaging. In this example, the symbol $\Delta \mathrm{x}$ is used to denote the change in DNA length that occurs when RecA is bound. $\mathbf{c}$ Multicolour total internal reflection microscopy (TIRFM) assays for visualizing RecA nucleation and growth on ssDNA ${ }^{88}$. Left panel, schematic of three-colour assay for visualizing AlexaFluor488-labelled single-stranded DNA-binding protein (SSB ${ }^{\mathrm{AF} 488}$, black), Cy3-labelled RecA (RecA ${ }^{C y} 3$, red) and fluorescein-labelled RecA (RecA ${ }^{f}$, green) on a surface-tethered ssDNA substrate. Filament nucleation is initiated by the addition of 
$\operatorname{Rec} \mathrm{A}^{\mathrm{Cy} 3}$, and subsequent growth of the filaments is monitored by injecting $\operatorname{Rec} \mathrm{A}^{\mathrm{f}}$ into the reactions. Right panels, TIRFM images showing nucleation and bidirectional growth of single RecA filaments. d | Schematic illustration and kymograph illustrating the assembly of a yeast DNA repair protein Rad51 filament using DNA curtains ${ }^{94}$. The ssDNA is first bound by fluorescently labelled replication protein A (GFP-RPA, green) and then chased with unlabelled Rad51 and ATP. Presynaptic complex assembly results in displacement of GFPRPA from the ssDNA, and the resulting Rad51 filament remains intact even after free Rad51 is flushed from the sample chamber. Removal of ATP from the buffer causes disassembly of the Rad51 filament as revealed by the rebinding of GFP-RPA. Part $\mathbf{b}$ is from REF. 87, Macmillan Publishers Limited, part $\mathbf{c}$ is from REF. 88, Macmillan Publishers Limited and part $\mathbf{d}$ is adapted with permission from REF. 94, Elsevier. a.u., arbitrary units. 


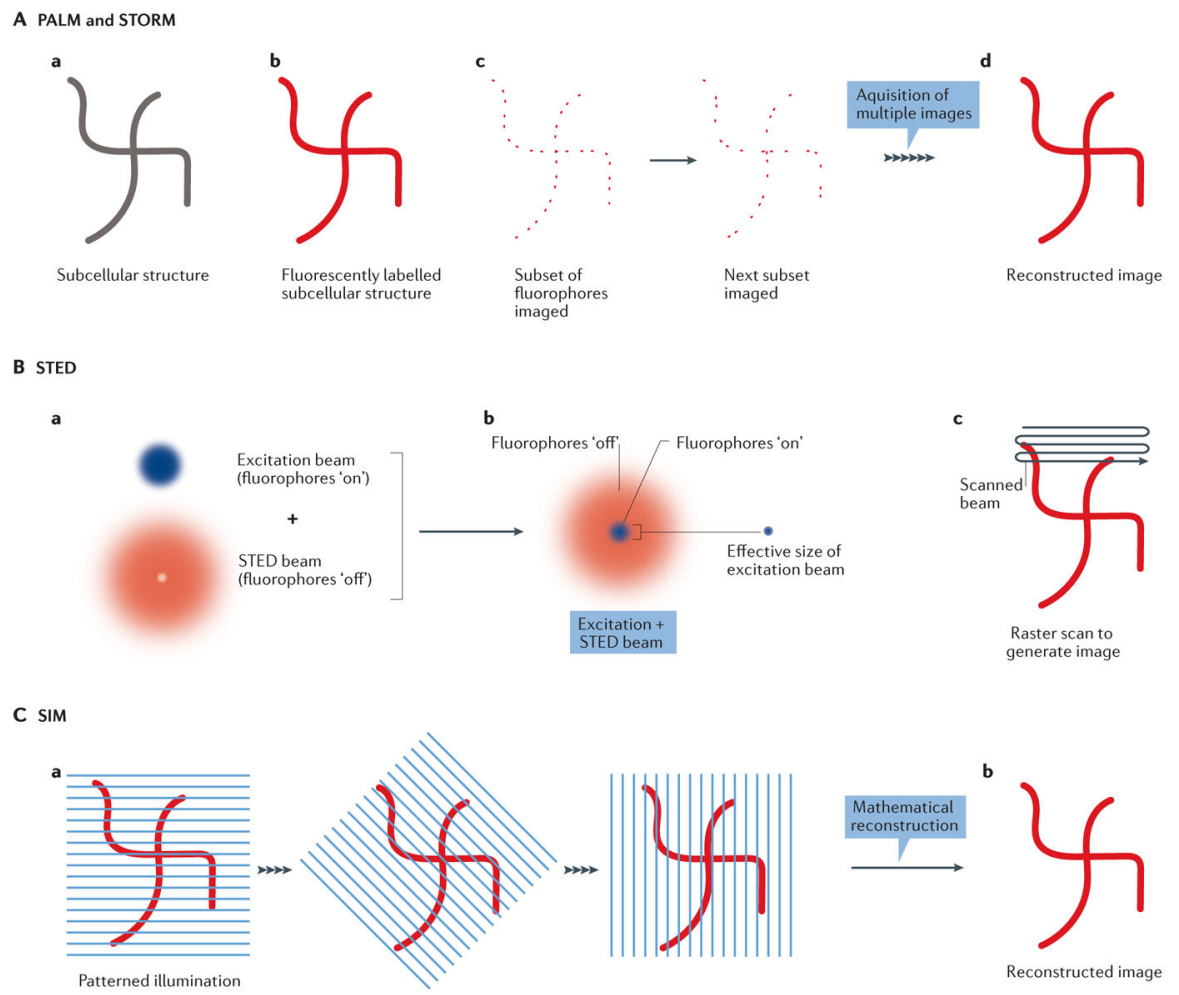

Figure 4. Super-resolution imaging methods

Part A shows a schematic representation of the principles allowing for super-resolution (SR) imaging by photo-activated localization microscopy (PALM) and stochastic optical reconstruction microscopy (STORM). A hypothetical subcellular structure (part Aa) is labelled with thousands of individual fluorophores (part Ab). PALM and STORM images are generated by collecting a series of images. Only a small subset of the total number of fluorophores present are illuminated in any given image, and a different subset of fluorophores is visible in each image (part Ac). The precise location of these spatially resolved fluorophores can be defined by fitting each observed 'spot' to a Gaussian (or similar) function. These cumulative data for all the 'spot' locations are then used to reconstruct the final SR image (part Ad). As shown in part $\mathbf{B}$, stimulated emission depletion (STED) microscopy uses two superimposed laser beams for sample illumination, depicted in red and blue (part Ba). One laser (blue) is used to excite (that is, turn 'on') the fluorophores conjugated to the subcellular structure of interest, and a second doughnut-shaped laser (red) is used to push the fluorophores back into an unexcited state (that is, 'off'). Superimposition of these two beams yields a very small region within the centre that can be used to excite fluorophores in the sample (part Bb), which can be scanned across a sample to yield an SR image (part Bc). In structured illumination microscopy (SIM), shown in part $\mathbf{C}$, a subset of fluorophores on the labelled sample is illuminated with a pattern of light, depicted as a blue 'striped' pattern (part Ca), and their positions are recorded. This pattern is then shifted through a series of different orientations, yielding images with encoded high-resolution information, and mathematical deconvolution of the resulting imaging data yields a highresolution image (part $\mathbf{C b}$ ). 

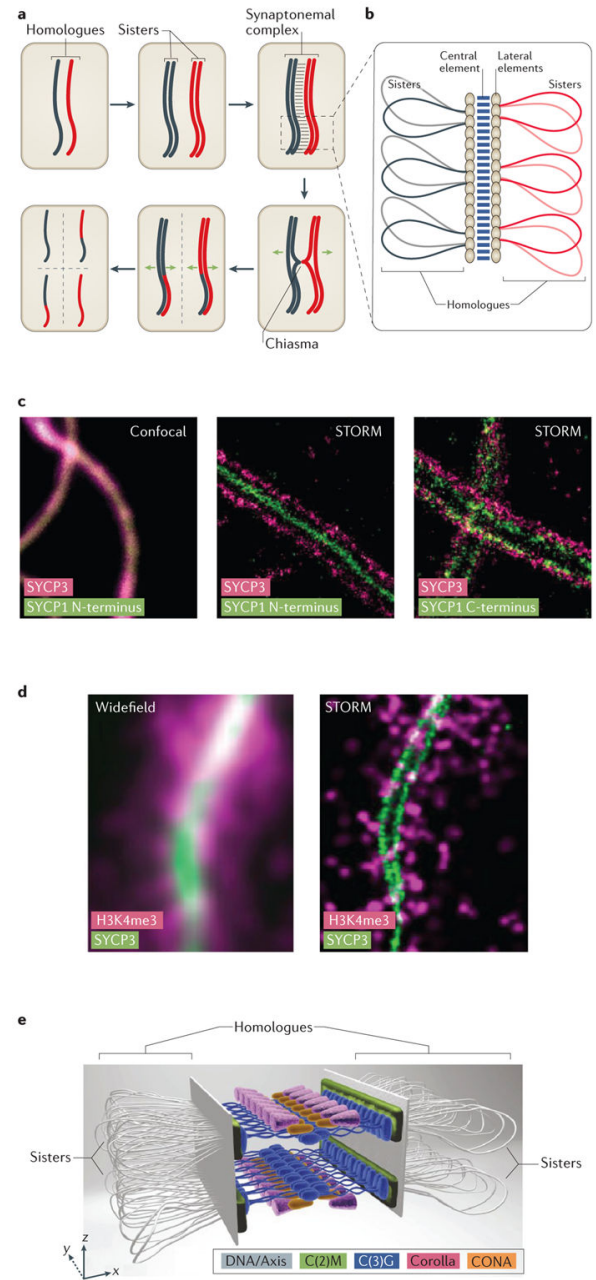

Figure 5. Super-resolution images of the synaptonemal complex

a A simplified overview of meiosis, which highlights DNA replication and segregation events involving a diploid cell with a single pair of homologous chromosomes (shown in red and black). Following an initial round of DNA replication, the resulting sister chromatids are linked together by cohesin (not depicted). Homologues are then aligned and linked along their lengths through formation of the synaptonemal complex, which comprises several meiosis-specific proteins ${ }^{133-136}$. Meiotic recombination takes place within the synaptonemal complex and results in crossover formation between homologues. The sites of crossover are called chiasma, which provide a physical linkage between homologues that is necessary to ensure attachment to the spindle apparatus (depicted as green arrows), allowing homologues to be separated to opposite poles of the cell. A second round of spindle formation allows segregation of the sister chromatids to yield haploid cells. b | Diagram of the synaptonemal complex highlighting the tethered-loop architecture with pairs of sister chromatids attached to the lateral elements, which are in turn held together by central element proteins. c | Confocal and stochastic optical reconstruction microscopy (STORM) images showing the relative locations of synaptonemal complex protein 3 (SYCP3) (magenta) and the SYCP1 Nterminus and C-terminus (green) within the synaptonemal complex ${ }^{137}$. SYCP3 co-localizes with the lateral elements, as does the C-terminus of SYCP1, whereas the N-terminus of 
SYCP1 is positioned towards the central element. Notably, these differences in protein spatial distributions cannot be distinguished in the confocal image. $\mathbf{d}$ | Widefield epifluorescence and STORM images showing the locations of SYCP3 (green) and H3K4me3-modified nucleosomes (magenta) ${ }^{138}$. In these images, the position of SYCP3 indicates the location of the lateral elements, and $\mathrm{H} 3 \mathrm{~K} 4 \mathrm{me} 3$ highlights what are anticipated to be transcriptionally active chromatin loops emanating outwards from the lateral elements. e | Illustration highlighting the proposed two-layered architecture of the synaptonemal complexes based on structured illumination microscopy images ${ }^{139}$. The key feature of this model is that the pairs of sister chromatid loops emanating outwards may be maintained in separate subcellular volumes, which could help minimize inter-sister recombination during meiosis and may help ensure homologue bias. Part $\mathbf{c}$ is adapted with permission from REF. 137, PNAS. Part $\mathbf{d}$ is adapted with permission from REF. 138, PNAS. Part $\mathbf{e}$ is reproduced with permission from Ryan Kramer. 


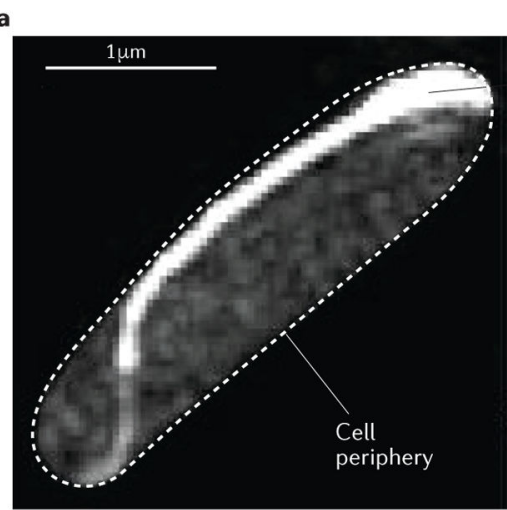

b

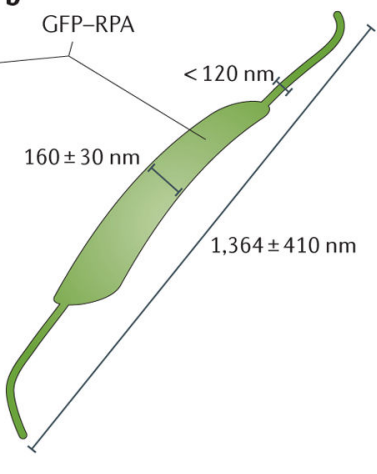

C

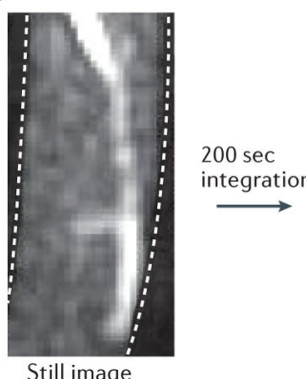

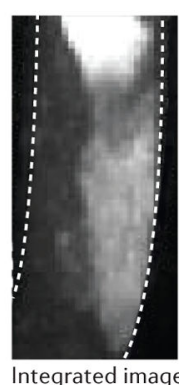

d

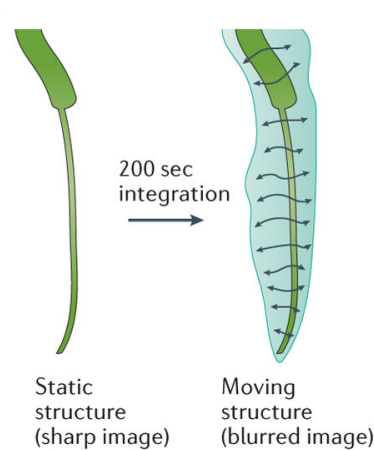

Figure 6. SIM imaging of recombination structures in living cells

a Structured illumination microscopy (SIM) image showing that protein RecA forms large structures, referred to as RecA bundles, during DNA repair in living Escherichia coli cells $^{147}$. b | A cartoon depiction of a typical RecA bundle, including the dimensions deduced from live-cell SIM imaging. $\mathbf{c}$ SIM images showing that the elongated tips of the RecA bundles are highly mobile. The panel on the left shows a single image, which appears stationary, and the panel on the right shows an image integrated over a period of 200 seconds; the 'blurring' observed in this image results from movement of the RecA bundle within the cell. $\mathbf{d} \mid$ Cartoon depiction of the RecA bundle images shown in part $\mathbf{c}$.

Arrowheads are used to highlight the movement of this structure within the cell. Parts $\mathbf{a}$ and c are from REF. 147, Macmillan Publishers Limited. 


\section{Table 1}

Functional grouping of E. coli, S. cerevisiae and human recombination proteins with associated human diseases *

\begin{tabular}{|c|c|c|c|c|}
\hline Function & E. coli & S. cerevisiae & Human & Disease \\
\hline Single-stranded DNA binding & $\mathrm{SSB}^{*}$ & $\mathrm{Rpa}^{*}$ & $\mathrm{RPA}^{*}$ & \\
\hline End resection & $\begin{array}{l}\cdot \operatorname{RecBCD}{ }^{*}, t \\
-\operatorname{Rec}{ }^{*}, t^{*} \\
-\operatorname{RecJ}{ }^{*}\end{array}$ & 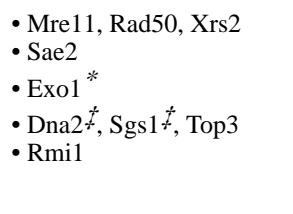 & 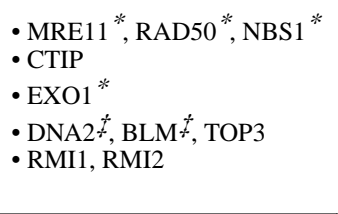 & $\begin{array}{l}\text { - Nijmegen breakage } \\
\text { syndrome } \\
\text { - Ataxia-telangiectasia-like } \\
\text { disorder } \\
\text { - Bloom syndrome } \\
\text { - Colorectal cancer } \\
\text { - Breast cancer }\end{array}$ \\
\hline Recombinases & $\operatorname{RecA}^{*}$ & $\operatorname{Rad} 51^{*}, \mathrm{Dmc1}{ }^{*}$ & RAD51 ${ }^{*}, \mathrm{DMC} 1^{*}$ & \\
\hline Recombination mediators & RecFOR $^{*}$ & $\begin{array}{l}\text { - } \operatorname{Rad} 52^{*} \\
\text { - } \operatorname{Rad} 55, \operatorname{Rad} 57 \\
\text { - Psy3, Csm2, Shu1, Shu2 }\end{array}$ & $\begin{array}{l}\text { - BRCA2, PALB2 } \\
\text { - RAD51B, RAD51C } \\
\text { - RAD51D } \\
\text { - XRCC2, XRCC3 } \\
\text { - RAD51D, XRCC2 }\end{array}$ & Breast cancer \\
\hline Helicases and anti-recombinases & $\begin{array}{l}\cdot \mathrm{UvrD}^{*}, t^{*} \\
\cdot \operatorname{Rep}^{*}, t\end{array}$ & $\begin{array}{l}\cdot \operatorname{Srs} 2^{*},{ }^{*} \\
\cdot \operatorname{Mph} 1^{*}\end{array}$ & $\begin{array}{l}\cdot \mathrm{RECQ} 5 *, \mathrm{FBH} 1{ }^{\hbar} \\
\cdot \text { FANCM }{ }^{\hbar} \\
\cdot \text { RTEL } 1^{\hbar}\end{array}$ & Breast cancer \\
\hline Branch migration & $\begin{array}{l}\cdot \operatorname{RuvA} \\
\cdot \operatorname{RuvB}^{*} \\
\cdot \operatorname{RecQ}^{*}, t^{t} \\
\cdot \operatorname{UvrD}{ }^{*}, t^{t}\end{array}$ & $\begin{array}{l}\cdot \operatorname{Sgs} 1^{*}-\mathrm{Top} 3-\operatorname{Rmi} 1 \\
\cdot \operatorname{Rad} 54^{*}, t^{*} \\
\cdot \operatorname{Rdh} 54^{*}, t^{*} \\
\cdot \operatorname{Mph} 1 *\end{array}$ & 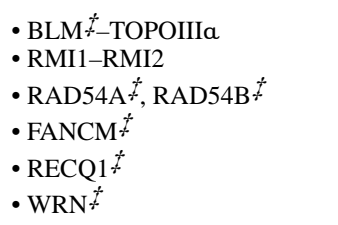 & $\begin{array}{l}\text { - Fanconi anaemia } \\
\text { - Hoyeraal-Hreidarsson } \\
\text { syndrome }\end{array}$ \\
\hline Holliday junction resolution & $\begin{array}{l}\text { - RecQ }{ }^{*}, t \\
\text { - TopoIII } \\
\text { - RuvC }\end{array}$ & $\begin{array}{l}\text { - Mus81-Mms4 } \\
\text { - Slx1-Slx4 } \\
\text { - Yen1 } \\
\text { - Rad1-Rad10 }\end{array}$ & $\begin{array}{l}\text { - MUS81-EME1 } \\
\cdot \text { SLX1-SLX4 } \\
\text { - GEN1 } \\
\cdot \text { XPF-ERCC1 }\end{array}$ & $\begin{array}{l}\text { - Bloom syndrome } \\
\text { - Fanconi anaemia }\end{array}$ \\
\hline
\end{tabular}

This is not intended to be a comprehensive list of proteins involved in homologous recombination but instead is intended to highlight a few examples of key proteins, including several proteins described in the main text. For more comprehensive discussion of these and other proteins, we refer readers to other recent reviews $2,3,13,15,16$.

Proteins that have been analysed by single-molecule or super-resolution methods.

${ }^{t}$ Motor proteins. 